\title{
Full orbital calculation scheme for materials with strongly correlated electrons
}

\author{
V. I. Anisimov, ${ }^{1}$ D. E. Kondakov, ${ }^{1}$ A. V. Kozhevnikov,,${ }^{1}$ I. A. Nekrasov,${ }^{1}$ Z. V. Pchelkina,${ }^{1}$ J. W. Allen, ${ }^{2}$ S.-K. Mo, ${ }^{2}$ \\ H.-D. Kim, ${ }^{3}$ P. Metcalf, ${ }^{4}$ S. Suga, ${ }^{5}$ A. Sekiyama,${ }^{6}$ G. Keller, ${ }^{7}$ I. Leonov, ${ }^{7}$ X. Ren ${ }^{7}$ and D. Vollhardt ${ }^{7}$ \\ ${ }^{1}$ Institute of Metal Physics, Russian Academy of Sciences-Ural Division, 620219 Yekaterinburg GSP-170, Russia \\ ${ }^{2}$ Randall Laboratory of Physics, University of Michigan, Ann Arbor, Michigan 48109, USA \\ ${ }^{3}$ Pohang Accelerator Laboratory, Pohang 790-784, Korea \\ ${ }^{4}$ Department of Physics, Purdue University, West Lafayette, Indiana 47907, USA \\ ${ }^{5}$ Division of Materials Physics, Graduate School of Engineering Science, Osaka University, Toyonaka, Osaka 560-8531, Japan \\ ${ }^{6}$ Graduate School of Engineering Science, Osaka University, Toyonaka, Osaka 560-8531, Japan \\ ${ }^{7}$ Theoretical Physics III, Center for Electronic Correlations and Magnetism, University of Augsburg, D-86135 Augsburg, Germany
}

(Received 24 July 2004; revised manuscript received 11 November 2004; published 21 March 2005)

\begin{abstract}
We propose a computational scheme for the ab initio calculation of Wannier functions (WFs) for correlated electronic materials. The full-orbital Hamiltonian $\hat{H}$ is projected into the WF subspace defined by the physically most relevant partially filled bands. The Hamiltonian $\hat{H}^{W F}$ obtained in this way, with interaction parameters calculated by constrained local-density approximation (LDA) for the Wannier orbitals, is used as an $a b$ initio setup of the correlation problem, which can then be solved by many-body techniques, e.g., dynamical mean-field theory (DMFT). In such calculations the matrix self-energy $\hat{\Sigma}(\varepsilon)$ is defined in WF basis which then can be converted back into the full-orbital Hilbert space to compute the full-orbital interacting Green function $G\left(\mathbf{r}, \mathbf{r}^{\prime}, \varepsilon\right)$. Using $G\left(\mathbf{r}, \mathbf{r}^{\prime}, \varepsilon\right)$ one can evaluate the charge density, modified by correlations, together with a new set of WFs, thus defining a fully self-consistent scheme. The Green function can also be used for the calculation of spectral, magnetic, and electronic properties of the system. Here we report the results obtained with this method for $\mathrm{SrVO}_{3}$ and $\mathrm{V}_{2} \mathrm{O}_{3}$. Comparisons are made with previous results obtained by the LDA+DMFT approach where the LDA density of states was used as input, and with new bulk-sensitive experimental spectra.
\end{abstract}

DOI: $10.1103 /$ PhysRevB.71.125119

PACS number(s): 71.27. $+\mathrm{a}, 71.30 .+\mathrm{h}$

\section{INTRODUCTION}

Model Hamiltonians used in the study of correlation effects in solids have a Coulomb interaction term in a sitecentered atomiclike orbital basis set which is not explicitly defined. When the correlated electrons are well localized, as, for example, $4 f$ states of rare-earth ions, atomic orbitals (or atomic sphere solutions like muffin-tin orbitals) are a good choice. However, the most interesting problems occur in the regime of metal-insulator transitions, where the states of interest become partially itinerant and rather extended. The error of using atomic orbitals is most severe in the case of materials with strong covalency effects, like late transitionmetal oxides, where partially filled bands are formed by the mixture of metallic $d$ orbitals and oxygen $p$ orbitals. For example, in high- $T_{c}$ cuprates correlated states have the symmetry of $\mathrm{Cu}-3 d x^{2}-y^{2}$ orbitals, but are actually Zhang-Rice singlets formed by the combination of oxygen $p$ states centered around the $\mathrm{Cu}$ ion and having $x^{2}-y^{2}$ symmetry.

In model calculations the problem of defining the correlated orbitals is not very important, because it only affects model parameter values, which in any case are considered fitting parameters. However, any attempt to construct an " $a b$ initio" calculation scheme requires an explicit definition of the basis set for the Coulomb interaction term. An important requirement for such a choice is that the orbitals must produce the partially filled bands where Coulomb correlations occur while preserving the localized, site-centered atomiclike form. These requirements are fulfilled for Wannier functions (WFs) $\left|W_{n}^{\mathbf{T}}\right\rangle$ defined as a Fourier transformation of the Bloch functions $\left|\psi_{n \mathbf{k}}\right\rangle \cdot{ }^{1}$ Here and below functions are labeled with band index $n$, lattice translation vector $\mathbf{T}$, and wave vector $\mathbf{k}$.

When there is more than one band crossing the Fermi level, WFs are not uniquely defined. Any k-dependent unitary transformation $\hat{U}^{(\mathbf{k})}$ of the set of Bloch functions $\left|\psi_{n \mathbf{k}}\right\rangle$ for these bands produces a new set which can be used for the calculation of WFs via Fourier transformation [Eq. (5), Sec. II A]. If one imposes the requirement that the WFs should have the symmetry of atomic orbitals, ${ }^{2,3}$ this unitary transformation is well defined. The explicit form of the WFs allows one to compute Coulomb interaction parameters in constrained local-density approximation (LDA) calculations.

In this way the parameters for the $a b$ initio many-body Hamiltonian (noninteracting Hamiltonian $\hat{H}^{W F}$ and Coulomb interaction) in the WF basis can be computed by any firstprinciple electronic structure calculation scheme [below we use the linear muffin-tin orbital (LMTO) method]. This Hamiltonian can then be further investigated by one of the methods developed in the many-body community. In the present work we use the dynamical mean-field theory (DMFT) ${ }^{4-7}$ Within DMFT, the effective impurity problem corresponding to the many-body Hamiltonian is solved by quantum Monte Carlo simulations (QMC). ${ }^{8}$ The DMFT part of the proposed calculation scheme is essentially the same as the one used in the recently developed LDA+DMFT approach $^{9}$ for the $a b$ initio investigations of correlated electron materials. ${ }^{10}$ However, here we propose a more general procedure to compute the Green function using the Hamiltonian matrix and an integral over the Brillouin zone instead of the Hilbert transform of the LDA density of states (DOS). 
This particular method allows one to avoid the uncontrollable errors occurring in the computation of the Green function using the Hilbert transform of the LDA DOS. Thus to obtain an insulating solution we need to cut off the long (metal-oxygen) hybridization tails of the DOS, renormalize it, and shift the Fermi energy to get an integer filling. In the present method we overcome the above-mentioned difficulties owing to the integer filling of Wannier orbitals. The result of the DMFT calculations is a local matrix self-energy $\hat{\Sigma}(\varepsilon)$ in the WF basis set $\left\{W_{n}\right\}$ which acts in the subspace of partially filled bands used for the construction of the WFs.

The paper is structured as follows. In Sec. II the details of our scheme are presented. In Sec. II A we describe the construction of WFs, as well as the ab initio Hamiltonian matrix within this basis set in terms of Bloch functions. In Sec. II B we propose a general method for the construction of WFs using the Green function $G\left(\mathbf{r}, \mathbf{r}^{\prime}, \varepsilon\right)$, which reduces to the results of Sec. II A in the noninteracting case. The reason for doing so is that the correlation effects can significantly renormalize the electronic states of the partially filled bands. Hence the WFs computed from noninteracting Bloch states are not an optimal choice for the basis set any more. In Sec. II C we discuss how to calculate within DMFT the local Green function with the input of the Hamiltonian matrix in the WF basis set instead of the LDA DOS (which is valid only in the case of degenerate bands). In Sec. II D we show that the matrix self-energy within the WF subspace, which is the solution of the correlation problem, can be transformed back into the full-orbital Hilbert space, thus enabling the computation of the full interacting Green function $G\left(\mathbf{r}, \mathbf{r}^{\prime}, \varepsilon\right)$. It can then be used to calculate the spectral, magnetic, and electronic properties of the system under investigation. In addition, to make the calculation scheme fully selfconsistent, one can employ the $G\left(\mathbf{r}, \mathbf{r}^{\prime}, \varepsilon\right)$ to calculate the charge density affected by correlations and thus the new LDA potential. Thereby the feedback from DMFT to LDA can be incorporated in a well-defined way. This is actually one of the great advantages of using the WF basis since in the LMTO basis the feedback from DMFT to LDA is essentially uncontrolled. In Sec. III the results for the electronic structure of the two vanadium oxides $\mathrm{SrVO}_{3}$ and $\mathrm{V}_{2} \mathrm{O}_{3}$ obtained by the method developed in this work are presented and compared with the previous calculations by the simpler methods and new bulk-sensitive spectra. Finally in Sec. IV we close this work with a conclusion.

\section{METHOD}

Let us consider the general case of the electronic structure problem. For the LDA Hamiltonian $\hat{H}$ we have a Hilbert space of eigenfunctions (Bloch states $\left.\left|\psi_{i \mathbf{k}}\right\rangle\right)$ with the basis set $\left|\phi_{\mu}\right\rangle$ defined by particular methods [e.g., LMTO, ${ }^{11}$ or linearized augmented plane waves (LAPW), ${ }^{12}$ etc.]. In this basis set the Hamiltonian operator is defined as

$$
\hat{H}=\sum_{\mu \nu}\left|\phi_{\mu}\right\rangle H_{\mu \nu}\left\langle\phi_{\nu}\right| .
$$

Here and later greek indices are used for full-orbital matrices.
If we consider a certain subset of the Hamiltonian eigenfunctions, for example Bloch states of partially filled bands $\left|\psi_{n \mathbf{k}}\right\rangle$, we can define a corresponding subspace in the total Hilbert space. The Hamiltonian matrix is diagonal in the Bloch states basis. However, physically more appealing is a basis set which has the form of site-centered atomic orbitals. That is, a set of WFs $\left|W_{n}^{\mathbf{T}}\right\rangle$ defined as the Fourier transformation of a certain linear combination of Bloch functions belonging to this subspace [see below Eq. (6)]. The Hamiltonian operator $\hat{H}^{W F}$ defined in this basis set is

$$
\hat{H}^{W F}=\sum_{n n^{\prime} \mathbf{T}}\left|W_{n}^{0}\right\rangle H_{n n^{\prime}}(\mathbf{T})\left\langle W_{n^{\prime}}^{\mathbf{T}}\right| .
$$

The total Hilbert space can be divided into a direct sum of the above introduced subspace (of partially filled Bloch states) and the subspace formed by all other states orthogonal to it. Those two subspaces are decoupled since they are the eigenfunctions corresponding to different eigenvalues. The Hamiltonian matrix in the WF basis (i.e., a collection of the bases of the specific subspaces) is block diagonal so that the matrix elements between different subspaces are zero. The block matrix $H_{n n^{\prime}}$ in Eq. (2) corresponding to the partially filled bands can be considered as a projection of the fullorbital Hamiltonian operator (1) onto the subspace defined by its WFs.

All this concerns the noninteracting (or LDA) Hamiltonian. To treat Coulomb correlations one also needs a definition of the localized orbitals where the electrons interact. WFs are a natural choice for such a definition. This choice leads to an important flexibility in the size of the basis set in the sense that the number of WFs can be changed by changing the set of Bloch bands considered. The simplest case is a set of partially filled bands, for example the $t_{2 g}$ bands of vanadium oxides. This is a physically justified approximation because the Coulomb interaction happens mainly between electrons (or holes) in the partially filled bands. If the problem to be solved concentrates on the excitation spectrum in a small energy window around the Fermi level, this basis set is sufficient. However, if the excitations to higher lying states (real or virtual) are also important, the set of Bloch bands used to construct the WFs need to be extended so that the Coulomb interaction will be treated in a larger Hilbert subspace.

Practically this means that the correlation problem is solved using a noninteracting few-orbital Hamiltonian $\hat{H}^{W F}$ [Eq. (2)] instead of the full Hilbert space Hamiltonian $\hat{H}$ [Eq. (1)]. The interaction matrix elements of the model Hamiltonian can be determined from constrained LDA calculations for the specific WF basis set (25).

Projecting the full orbital Hilbert space Hamiltonian $\hat{H}$ [Eq. (1)] onto the subspace of the partially filled bands gives us a few-orbital Hamiltonian $\hat{H}^{W F}$ [Eq. (2)]. This significantly decreases the complexity of the correlation problem, thus permitting its explicit solution. The many-body problem with a local intraorbital Coulomb interaction (Hubbard interaction) then leads to a local matrix self-energy $\hat{\Sigma}^{W F}(\varepsilon)$ which 
is naturally defined in the basis of WFs centered on the same site: ${ }^{13}$

$$
\hat{\Sigma}^{W F}(\varepsilon)=\sum_{n n^{\prime}}\left|W_{n}^{0}\right\rangle \Sigma_{n n^{\prime}}(\varepsilon)\left\langle W_{n^{\prime}}^{0}\right| .
$$

We note that, in contrast to other "basis-reducing" methods, the information about the states corresponding to the bands below and above the projected ones is not lost. In fact, the information is stored in the k-dependent projection matrix between the full orbital basis set and the orthonormalized WFs (16). The definition (3) allows one to convert the matrix self-energy $\Sigma_{n n^{\prime}}(\varepsilon)$ back to the full Hilbert space basis set (Sec. II D). With this the interacting Green function can also be calculated in the full-orbital Hilbert space (Sec. II B)

\section{A. Definition and construction of Wannier functions}

The concept of WFs has a very important place in the electron theory in solids since its first introduction in 1937 by Wannier. ${ }^{1}$ WFs are the Fourier transformation of Bloch states $\left|\psi_{i \mathbf{k}}\right\rangle$,

$$
\left|W_{i}^{\mathbf{T}}\right\rangle=\frac{1}{\sqrt{N}} \sum_{\mathbf{k}} e^{-i \mathbf{k} \mathbf{T}}\left|\psi_{i \mathbf{k}}\right\rangle,
$$

where $N$ is the number of discrete $\mathbf{k}$ points in the first Brillouin zone (or, the number of cells in the crystal). These extremely convenient orthogonal functions were widely investigated in the seventies. ${ }^{14}$ The strongly localized nature of the WFs together with all advantages of the atomic functions makes them a very useful tool where the atomic character of the electrons is highlighted. Thus, using the WF method, significant progress was achieved in the fields of narrowband superconductors, disordered systems, solid surfaces, etc. Several methods for calculating WFs for single and multiple bands in periodic crystals and their generalization to nonperiodic systems were proposed. The problem of nonunique definition of WFs in these methods was resolved by an iterative optimization of trial functions which have the same real and point-group symmetry properties as WFs. Among these methods, there are the variational Koster-Parzen principle ${ }^{15,16}$ which was generalized by Kohn, ${ }^{20-25}$ the general pseudopotential formalism proposed by Anderson, ${ }^{26}$ and the projection operator formalism by Cloizeaux. ${ }^{27-29}$ However, all these computational schemes are restricted to simple band structures.

Wannier functions are not uniquely defined because for a certain set of bands any orthogonal linear combination of Bloch functions $\left|\psi_{i \mathbf{k}}\right\rangle$ can be used in Eq. (4). In general it means that the freedom of choice of Wannier functions corresponds to the freedom of choice of a unitary transformation matrix $U_{j i}^{(\mathbf{k})}$ for corresponding Bloch functions:2

$$
\left|\tilde{\psi}_{i \mathbf{k}}\right\rangle=\sum_{j} U_{j i}^{(\mathbf{k})}\left|\psi_{j \mathbf{k}}\right\rangle
$$

The resulting Bloch function $\left|\widetilde{\psi}_{i \mathbf{k}}\right\rangle$ will generally not be an eigenfunction of the Hamiltonian but has the meaning of a Bloch sum of Wannier functions [see below $\left|\widetilde{W}_{n \mathbf{k}}\right\rangle$ in Eq.
(6)]. There is no rigorous way to define $U_{j i}^{(\mathbf{k})}$. This calls for an additional restriction on the properties of WFs. Among others, Marzari and Vanderbilt ${ }^{2}$ proposed the condition of maximum localization for WFs, resulting in a variational procedure to calculate $U_{j i}^{(\mathbf{k})}$. To get a good initial guess the authors of Ref. 2 proposed choosing a set of localized trial orbitals $\left|\phi_{n}\right\rangle$ and projecting them onto the Bloch functions $\left|\psi_{i \mathbf{k}}\right\rangle$. It was found that this starting guess is usually quite good. This fact later led to the simplified calculating scheme proposed in Ref. 3 where the variational procedure was abandoned and the result of the aforementioned projection was considered as the final step. The approach of Ref. 2 has recently been used for the investigation of the row of $3 d$ transition metals $(\mathrm{Fe}$, $\mathrm{Co}, \mathrm{Ni}$, and $\mathrm{Cu}$ ) within the simplest many-body approximation, namely the unscreened Hartree-Fock approximation. ${ }^{30}$

Another possibility to construct WFs was recently developed by Andersen et al. ${ }^{31}$ They proposed the $N$ th-order muffin-tin orbital (NMTO) scheme in which Wannier-like low-energy MTOs can be designed a priori. Using a different implementation of the LDA+DMFT approach they performed an investigation of the Mott transition in orthorhombic $3 d^{1}$ perovskites. ${ }^{34}$ In this approach a realistic Hamiltonian constructed with Wannier orbitals (on symmetrically orthonormalized NMTOs) was solved by DMFT, including the nondiagonal part of the on-site self-energy.

Our projection procedure works as follows. First of all one needs to identify the physically relevant bands which will then be projected onto a WF basis. For example, in perovskites one usually takes the partially filled $d$ shell or some particular $d$ bands of transition metals, since they are mainly responsible for the physical properties of the system. ${ }^{10}$ These orbitals are well separated and are, in our approach, easily extracted from the full orbital space as will be shown later. Moreover, the projection method is applicable even in the case where the bands of interest differ and are strongly hybridized (for example, $\mathrm{Cu}-3 d$ and $\mathrm{O}-2 p$ states in high- $T_{c}$ superconductors ${ }^{32}$ ).

To project bands of particular symmetry onto the WFs basis one can select either the band indices of the corresponding Bloch functions $\left(N_{1}, \ldots, N_{2}\right)$, or choose the energy interval $\left(E_{1}, E_{2}\right)$ in which the bands are located. Nonorthogonalized WFs in reciprocal space $\left|\tilde{W}_{n \mathbf{k}}\right\rangle$ are then the projection of the set of site-centered atomiclike trial orbitals $\left|\phi_{n}\right\rangle$ on the Bloch functions $\left|\psi_{i \mathbf{k}}\right\rangle$ of the chosen bands [band indices $N_{1}$ to $N_{2}$, energy interval $\left.\left(E_{1}, E_{2}\right)\right]$ :

$$
\left|\tilde{W}_{n \mathbf{k}}\right\rangle \equiv \sum_{i=N_{1}}^{N_{2}}\left|\psi_{i \mathbf{k}}\right\rangle\left\langle\psi_{i \mathbf{k}} \mid \phi_{n}\right\rangle=\sum_{i\left(E_{1}<\varepsilon_{i}(\mathbf{k})<E_{2}\right)}\left|\psi_{i \mathbf{k}}\right\rangle\left\langle\psi_{i \mathbf{k}} \mid \phi_{n}\right\rangle .
$$

Then the real space WFs $\left|\tilde{W}_{n}^{\mathbf{T}}\right\rangle$ are given by

$$
\left|\tilde{W}_{n}^{\mathbf{T}}\right\rangle=\frac{1}{\sqrt{N}} \sum_{\mathbf{k}} e^{-i \mathbf{k} \mathbf{T}}\left|\tilde{W}_{n \mathbf{k}}\right\rangle .
$$

In the present work the trial orbitals $\left|\phi_{n}\right\rangle$ are LMTOs. Note that in the multiband case a WF in reciprocal space $\left|\widetilde{W}_{n \mathbf{k}}\right\rangle$ does not coincide with the Bloch function $\left|\psi_{n \mathbf{k}}\right\rangle$ due to the 
summation over band index $i$ in Eq. (6). One can consider them as Bloch sums of WFs analogous to the basis function Bloch sums $\phi_{j}^{\mathbf{k}}(\mathbf{r})$ [Eq. (9)].

The coefficients $\left\langle\psi_{i \mathrm{k}} \mid \phi_{n}\right\rangle$ in Eq. (6) define (after orthonormalization) the unitary transformation matrix $U_{j i}^{(\mathbf{k})}$ in Eq. (5). However, the projection procedure defined in Eq. (6) is more general than the unitary transformation (5). Namely, the number of bands $\left(N_{2}-N_{1}+1\right)$ can be larger than the number of trial functions. In this case the projection (6) will produce $N$ new functions $\left|\widetilde{W}_{n \mathbf{k}}\right\rangle$ which define a certain subspace of the original $\left(N_{2}-N_{1}+1\right)$-dimensional space. This subspace will have the symmetry of the set of trial functions. In the next subsection we propose a way to determine WFs from the Green function of the system (28) rather than from a set of Bloch states as in Eq. (6). In this alternative projection procedure, trial functions are projected onto the subspace defined by the Green function in a certain energy interval.

The Bloch functions in the LMTO basis (or any other atomic orbital-like basis set) are defined as

$$
\left|\psi_{i \mathbf{k}}\right\rangle=\sum_{\mu} c_{\mu i}^{\mathbf{k}}\left|\phi_{\mu}^{\mathbf{k}}\right\rangle
$$

where $\mu$ is the combined index representing $q \operatorname{lm}$ ( $q$ is the atomic number in the unit cell, $l m$ are orbital and magnetic quantum numbers), $\phi_{\mu}^{\mathbf{k}}(\mathbf{r})$ are the Bloch sums of the basis orbitals $\phi_{\mu}(\mathbf{r}-\mathbf{T})$,

$$
\phi_{\mu}^{\mathbf{k}}(\mathbf{r})=\frac{1}{\sqrt{N}} \sum_{\mathbf{T}} e^{i \mathbf{k} \mathbf{T}} \phi_{\mu}(\mathbf{r}-\mathbf{T}),
$$

and the coefficients have the property

$$
c_{\mu i}^{\mathbf{k}}=\left\langle\phi_{\mu} \mid \psi_{i \mathbf{k}}\right\rangle .
$$

If $n$ in $\left|\phi_{n}\right\rangle$ corresponds to the particular qlm combination (in other words $\left|\phi_{n}\right\rangle$ is an orthogonal LMTO basis set orbital), then $\left\langle\psi_{i \mathbf{k}} \mid \phi_{n}\right\rangle=c_{n i}^{\mathbf{k}^{*}}$, and hence

$$
\left|\tilde{W}_{n \mathbf{k}}\right\rangle=\sum_{i=N_{1}}^{N_{2}}\left|\psi_{i \mathbf{k}}\right\rangle c_{n i}^{\mathbf{k}^{*}}=\sum_{i=N_{1}}^{N_{2}} \sum_{\mu} c_{\mu i}^{\mathbf{k}} c_{n i}^{\mathbf{k}^{*}}\left|\phi_{\mu}^{\mathbf{k}}\right\rangle=\sum_{\mu} \widetilde{b}_{\mu n}^{\mathbf{k}}\left|\phi_{\mu}^{\mathbf{k}}\right\rangle,
$$

with

$$
\widetilde{b}_{\mu n}^{\mathbf{k}} \equiv \sum_{i=N_{1}}^{N_{2}} c_{\mu i}^{\mathbf{k}} c_{n i}^{\mathbf{k}^{*}} .
$$

For a nonorthogonal basis set, see Appendix C.

In order to orthonormalize the WFs (11) one needs to calculate the overlap matrix $O_{n n^{\prime}}(\mathbf{k})$,

$$
O_{n n^{\prime}}(\mathbf{k}) \equiv\left\langle\tilde{W}_{n \mathbf{k}} \mid \widetilde{W}_{n^{\prime} \mathbf{k}}\right\rangle=\sum_{i=N_{1}}^{N_{2}} c_{n i}^{\mathbf{k}} c_{n^{\prime} i}^{\mathbf{k}^{*}},
$$

and its inverse square root $S_{n n^{\prime}}(\mathbf{k})$ which is defined as

$$
S_{n n^{\prime}}(\mathbf{k}) \equiv O_{n n^{\prime}}^{-1 / 2}(\mathbf{k}) .
$$

In the derivation of Eq. (13) the orthogonality of Bloch states $\left\langle\psi_{n \mathbf{k}} \mid \psi_{n^{\prime} \mathbf{k}}\right\rangle=\delta_{n n^{\prime}}$ was used.
From Eqs. (11) and (14), the orthonormalized WFs in $\mathbf{k}$ space $\left|W_{n \mathbf{k}}\right\rangle$ can be obtained as

$$
\left|W_{n \mathbf{k}}\right\rangle=\sum_{n^{\prime}} S_{n n^{\prime}}(\mathbf{k})\left|\tilde{W}_{n^{\prime} \mathbf{k}}\right\rangle=\sum_{i=N_{1}}^{N_{2}}\left|\psi_{i \mathbf{k}}\right\rangle \bar{c}_{n i}^{\mathbf{k}^{*}}=\sum_{\mu} b_{\mu n}^{\mathbf{k}}\left|\phi_{\mu}^{\mathbf{k}}\right\rangle,
$$

with

$$
\begin{gathered}
\bar{c}_{n i}^{\mathbf{k}^{*}} \equiv\left\langle\psi_{i \mathbf{k}} \mid W_{n \mathbf{k}}\right\rangle=\sum_{n^{\prime}} S_{n n^{\prime}}(\mathbf{k}) c_{n^{\prime} i}^{\mathbf{k}^{*}}, \\
b_{\mu n}^{\mathbf{k}} \equiv\left\langle\phi_{\mu}^{\mathbf{k}} \mid W_{n \mathbf{k}}\right\rangle=\sum_{i=N_{1}}^{N_{2}} c_{\mu i}^{\mathbf{k}} \mathbf{k}_{n i}^{\mathbf{k}^{*}} .
\end{gathered}
$$

The real space site-centered WFs at the origin $\left|W_{n}^{0}\right\rangle$ are given by the Fourier transform of $\left|W_{n \mathbf{k}}\right\rangle$ with $\mathbf{T}=0$. From Eqs. (15) and (9) one finds

$$
\begin{aligned}
W_{n}(\mathbf{r}) & =\frac{1}{\sqrt{N}} \sum_{\mathbf{k}}\left\langle\mathbf{r} \mid W_{n \mathbf{k}}\right\rangle=\sum_{\mathbf{T}, \mu}\left(\frac{1}{N} \sum_{\mathbf{k}} e^{i \mathbf{k} T} b_{\mu n}^{\mathbf{k}}\right) \phi_{\mu}(\mathbf{r}-\mathbf{T}) \\
& =\sum_{\mathbf{T}, \mu} w^{\prime}(n, \mu, \mathbf{T}) \phi_{\mu}(\mathbf{r}-\mathbf{T})=\sum_{s} w(n, s) \phi_{\alpha(s)}\left(\mathbf{r}-\mathbf{T}_{s}\right),
\end{aligned}
$$

where $w^{\prime}$ and $w$ are the expansion coefficients of WF in terms of the corresponding LMTO orbitals, in particular,

$$
w(n, s)=\frac{1}{N} \sum_{\mathbf{k}} e^{i \mathbf{k} T_{s}} b_{\alpha(s) n}^{\mathbf{k}} .
$$

Here $s$ is an index counting the orbitals of the neighboring cluster for the atom where orbital $n$ is centered $\left[\mathbf{T}_{s}\right.$ is the corresponding translation vector, $\alpha(s)$ is a combined qlm index]. The explicit form of the real-space WF (18) can be used to produce, e.g., shapes of chemical bonds.

For other applications only the matrix elements of the various operators in the basis of WF (15) are needed. From Eqs. (15), (16), and (18) the matrix elements of the Hamiltonian $\hat{H}^{W F}$ in the basis of WF in real space where both orbitals are in the same unit cell are

$$
\begin{aligned}
H_{n n^{\prime}}^{W F}(\mathbf{0}) & =\left\langle W_{n}^{\mathbf{0}}\left|\left(\frac{1}{N} \sum_{\mathbf{k}} \sum_{i=N_{1}}^{N_{2}}\left|\psi_{i \mathbf{k}}\right\rangle \epsilon_{i}(\mathbf{k})\left\langle\psi_{i \mathbf{k}}\right|\right)\right| W_{n^{\prime}}^{\mathbf{0}}\right\rangle \\
& =\frac{1}{N} \sum_{\mathbf{k}} \sum_{i=N_{1}}^{N_{2}} \bar{c}_{n i}^{\mathbf{k}} \bar{c}_{n^{\prime} i} \mathbf{k}_{i}^{*} \epsilon_{i}(\mathbf{k}) .
\end{aligned}
$$

$\epsilon_{i}(\mathbf{k})$ is the eigenvalue for a particular band.

If, on the other hand, one of the orbitals corresponds to the WF for the atom $n^{\prime}$ shifted from its position in the primary unit cell by a translation vector $\mathbf{T}$, then the corresponding Hamiltonian matrix element is

$$
H_{n n^{\prime}}^{W F}(\mathbf{T})=\left\langle W_{n}^{\mathbf{0}}|\hat{H}| W_{n^{\prime}}^{\mathbf{T}}\right\rangle=\frac{1}{N} \sum_{\mathbf{k}} \sum_{i=N_{1}}^{N_{2}} \bar{c}_{n i}^{\mathbf{k}} \bar{c}_{n^{\prime} i}^{\mathbf{k}^{*}} \epsilon_{i}(\mathbf{k}) e^{-i \mathbf{k} \mathbf{T}} .
$$


Matrix elements of the density-matrix operator (occupation matrix $Q_{n m}^{W F}$ ) in the basis of WFs can be calculated as

$$
\begin{aligned}
Q_{n n^{\prime}}^{W F}(\mathbf{T}) & =\left\langle W_{n}^{0}\left|\left(\frac{1}{N} \sum_{\mathbf{k}} \sum_{i=N_{1}}^{N_{2}}\left|\psi_{i \mathbf{k}}\right\rangle \theta\left(E_{f}-\epsilon_{i}(\mathbf{k})\right)\left\langle\psi_{i \mathbf{k}}\right|\right)\right| W_{n^{\prime}}^{\mathbf{T}}\right\rangle \\
& =\frac{1}{N} \sum_{\mathbf{k}} \sum_{i=N_{1}}^{N_{2}} \bar{c}_{n i}^{\mathbf{k}} \bar{c}_{n^{\prime} i}^{*} \theta\left[E_{f}-\epsilon_{i}(\mathbf{k})\right] e^{-i \mathbf{k} \mathbf{T}} ;
\end{aligned}
$$

$\theta(x)$ is the step function, $E_{f}$ is the Fermi energy.

Finally, the matrix elements of the Hamiltonian $\hat{H}^{W F}$ in reciprocal space are

$$
\begin{aligned}
H_{n n^{\prime}}^{W F}(\mathbf{k}) & =\left\langle W_{n \mathbf{k}}\left|\left(\frac{1}{N} \sum_{\mathbf{k}^{\prime}} \sum_{i=N_{1}}^{N_{2}}\left|\psi_{i \mathbf{k}^{\prime}}\right\rangle \epsilon_{i}\left(\mathbf{k}^{\prime}\right)\left\langle\psi_{i \mathbf{k}^{\prime}}\right|\right)\right| W_{n^{\prime} \mathbf{k}}\right\rangle \\
& =\sum_{i=N_{1}}^{N_{2}} \bar{c}_{n i}^{\mathbf{k}} \bar{c}_{n^{\prime} i}^{\mathbf{k}^{*}} \epsilon_{i}(\mathbf{k}) .
\end{aligned}
$$

Equation (23) is valid only if the WFs are computed by Eqs. (15)-(17). If the WFs were obtained in one calculation and then used to compute the Hamiltonian matrix in another (as is the case for the WFs (28) in the Green-function formalism (see Sec. II B) then Eq. (23) is not valid any more and the general expression must be used:

$$
H_{n n^{\prime}}^{W F}(\mathbf{k})=\sum_{i=N_{1}}^{N_{2}} \epsilon_{i}(\mathbf{k}) \sum_{\mu} b_{\mu n}^{\mathbf{k}^{*}} c_{\mu i}^{\mathbf{k}} \sum_{\nu} b_{\nu n^{\prime}}^{\mathbf{k}} c_{\nu i}^{\mathbf{k}^{*}} .
$$

Thus the transformation from LMTO to the WF basis set is defined by the explicit form of WFs (15) and (17), and by the expressions of the matrix elements of the Hamiltonian and density matrix operators in WF basis (20) and (22). The back transformation from the WF to the LMTO basis can also be defined using Eq. (15) (see Sec. II D).

Finally, the Coulomb matrix element $U$ needs to be calculated in the same WF basis. This requires a method similar to the constrained LDA, ${ }^{33}$ but now for WFs. To this end the WF energy (20) is computed as a function of its occupancy (22) for a given WF $n$. Then the corresponding Coulomb interaction parameter $U_{n}$ in the WF basis is given by

$$
U_{n} \equiv \frac{d H_{n n}^{W F}(\mathbf{0})}{d Q_{n n}^{W F}(\mathbf{0})}
$$

As one can see, $U_{n}$ depends on the WFs via Eqs. (20) and (22). Once the WFs have been recalculated (for example, in some self-consistent loop) the interaction has to be recalculated as well.

WFs not only contain $d$ orbitals but also states which are usually considered delocalized, such as $\mathrm{O}-2 p$ orbitals. The question is then: which ones are the localized functions that describe the interacting electrons? In our definition these are WFs describing the partially occupied bands and not pure $d$ orbitals. The corresponding Coulomb interaction strength calculated in constrained LDA will give values of $U$ for Wannier functions smaller than the corresponding value for pure $d$ orbitals due to the admixture of $\mathrm{O}-2 p$ states.

\section{B. Wannier functions in the Green-function formalism}

In many-body theory the system is usually not described by Bloch functions $\left|\psi_{i \mathbf{k}}\right\rangle$ [Eq. (8)] and their energies $\epsilon_{i}(\mathbf{k})$ but by the Green function

$$
G\left(\mathbf{r}, \mathbf{r}^{\prime}, \varepsilon\right)=\frac{1}{N} \sum_{\mathbf{k}} G^{\mathbf{k}}\left(\mathbf{r}, \mathbf{r}^{\prime}, \varepsilon\right)=\frac{1}{N} \sum_{\mathbf{k}} \sum_{\mu \nu} \phi_{\mu}^{\mathbf{k}}(\mathbf{r}) G_{\mu \nu}^{\mathbf{k}}(\varepsilon) \phi_{\nu}^{* \mathbf{k}}\left(\mathbf{r}^{\prime}\right) .
$$

The matrix Green function $G_{\mu \nu}^{\mathbf{k}}(\varepsilon)$ is defined via the noninteracting Hamiltonian matrix $H_{\mu \nu}(\mathbf{k})$ and the matrix selfenergy $\Sigma_{\mu \nu}^{\mathbf{k}}(\varepsilon)[$ Eq. (39)] as

$$
G_{\mu \nu}^{\mathbf{k}}(\varepsilon)=[\varepsilon-\hat{H}(\mathbf{k})-\hat{\Sigma}(\varepsilon, \mathbf{k})+i \eta]_{\mu \nu}^{-1} .
$$

We define the nonorthonormalized WF obtained by projecting the trial orbital $\phi_{n}(\mathbf{r})$ on the Hilbert subspace defined by the Green function (26) in the energy interval $\left(E_{1}, E_{2}\right)$, namely,

$$
\begin{aligned}
\widetilde{W}_{n \mathbf{k}}(\mathbf{r}) & =-\frac{1}{\pi} \operatorname{Im} \int_{E_{1}}^{E_{2}} d \varepsilon \int d \mathbf{r}^{\prime} G^{\mathbf{k}}\left(\mathbf{r}, \mathbf{r}^{\prime}, \varepsilon\right) \phi_{n}^{\mathbf{k}}\left(\mathbf{r}^{\prime}\right) \\
& =\sum_{\mu} \widetilde{b}_{\mu n}^{\mathbf{k}} \phi_{\mu}^{\mathbf{k}}(\mathbf{r})
\end{aligned}
$$

and

$$
\widetilde{b}_{\mu n}^{\mathbf{k}} \equiv-\frac{1}{\pi} \operatorname{Im} \int_{E_{1}}^{E_{2}} d \varepsilon G_{\mu n}^{\mathbf{k}}(\varepsilon) .
$$

In the noninteracting case, the self-energy $\hat{\Sigma}(\varepsilon, \mathbf{k})$ is absent, and hence we have

$$
G_{\mu \nu}^{\mathbf{k}}(\varepsilon)=\sum_{i} \frac{c_{\mu i}^{\mathbf{k}} \mathbf{c}_{\nu i}^{\mathbf{k}^{*}}}{\varepsilon-\epsilon_{i}(\mathbf{k})+i \eta},
$$

where $c_{\mu i}^{\mathbf{k}}$ are the eigenvectors (10), and $\epsilon_{i}(\mathbf{k})$ are the eigenvalues of $\hat{H}(\mathbf{k})$. Thus $\widetilde{b}_{\mu n}^{\mathbf{k}}$ in Eq. (29) becomes

$$
\tilde{b}_{\mu n}^{\mathbf{k}}=\sum_{i=N_{1}}^{N_{2}} c_{\mu i}^{\mathbf{k}} c_{n i}^{\mathbf{k}^{*}}
$$

where $N_{1}, N_{2}$ are the band numbers which correspond to the energy interval $\left(E_{1}, E_{2}\right)$. Since this recovers the result of Eq. (12), we demonstrated that our general definition of WFs (28) via Green functions reduces to that in terms of Bloch functions (11) in Sec. II A.

To orthonormalize $\widetilde{W}_{n \mathbf{k}}(\mathbf{r})$ defined in Eq. (28), one can just follow the orthonormalizing procedure made in Sec. II A [Eqs. (13)-(17)], which will not be repeated here. But it should be pointed out that in the Green-function formalism the overlap matrix $O_{n n^{\prime}}(\mathbf{k})$ is defined as

$$
O_{n n^{\prime}}(\mathbf{k})=\left\langle\widetilde{W}_{n \mathbf{k}} \mid \widetilde{W}_{n^{\prime} \mathbf{k}}\right\rangle=\sum_{\mu} \widetilde{b}_{\mu n}^{\mathbf{k}^{*}} \widetilde{b}_{\mu n^{\prime}}^{\mathbf{k}}
$$

The occupancy matrix in the orthogonalized WF basis (28) is defined as 


$$
\begin{aligned}
Q_{n n^{\prime}}(\mathbf{T})= & -\frac{1}{\pi} \operatorname{Im} \int_{-\infty}^{E_{F}} d \varepsilon \iint d \mathbf{r} d \mathbf{r}^{\prime} \frac{1}{N} \sum_{\mathbf{k}} W_{n \mathbf{k}}^{*}(\mathbf{r}) \\
& \times G^{\mathbf{k}}\left(\mathbf{r}, \mathbf{r}^{\prime}, \varepsilon\right) W_{n^{\prime} \mathbf{k}}\left(\mathbf{r}^{\prime}\right) e^{-i \mathbf{k} \mathbf{T}} .
\end{aligned}
$$

By using Eq. (26) and orthogonalized Eq. (28), one finds

$$
Q_{n n^{\prime}}(\mathbf{T})=\frac{1}{N} \sum_{\mathbf{k}} \sum_{\mu \nu} b_{\mu n}^{\mathbf{k}^{*}} b_{\nu n^{\prime}}^{\mathbf{k}} Q_{\mu \nu}^{\mathbf{k}} e^{-i \mathbf{k} \mathbf{T}}
$$

with

$$
Q_{\mu \nu}^{\mathbf{k}}=-\frac{1}{\pi} \operatorname{Im} \int_{-\infty}^{E_{F}} d \varepsilon G_{\mu \nu}^{\mathbf{k}}(\varepsilon) .
$$

The energy matrix can be defined similarly (except that the integral over energy is calculated in the $\left(E_{1}, E_{2}\right)$ interval where the corresponding WFs are defined) as

$$
\begin{aligned}
E_{n n^{\prime}}(\mathbf{T})= & -\frac{1}{\pi} \operatorname{Im} \int_{E_{1}}^{E_{2}} \varepsilon d \varepsilon \iint d \mathbf{r} d \mathbf{r}^{\prime} \frac{1}{N} \sum_{\mathbf{k}} W_{n \mathbf{k}}^{*}(\mathbf{r}) \\
& \times G^{\mathbf{k}}\left(\mathbf{r}, \mathbf{r}^{\prime}, \varepsilon\right) W_{n^{\prime} \mathbf{k}}\left(\mathbf{r}^{\prime}\right) e^{-i \mathbf{k} \mathbf{T}} \\
= & \frac{1}{N} \sum_{\mathbf{k}} \sum_{\mu \nu} b_{\mu n}^{\mathbf{k}^{*}} b_{\nu n^{\prime}}^{\mathbf{k}} E_{\mu \nu}^{\mathbf{k}} e^{-i \mathbf{k} \mathbf{T}},
\end{aligned}
$$

with

$$
E_{\mu \nu}^{\mathbf{k}}=-\frac{1}{\pi} \operatorname{Im} \int_{E_{1}}^{E_{2}} \varepsilon d \varepsilon G_{\mu \nu}^{\mathbf{k}}(\varepsilon) .
$$

While Eq. (35) looks similar to the noninteracting Hamiltonian in WFs basis (21), it includes correlations via $\hat{\Sigma}(\varepsilon)$ in Eq. (27) and hence is interacting.

\section{DMFT in the Wannier function formalism}

In the previous subsection we showed [Eqs. (26)-(28)] that the matrix self-energy is needed to construct the WFs in terms of the full interacting Green function. The DMFT (Refs. 4-6) was recently found to be a powerful tool to numerically solve multiband Hubbard models. To define parameters of the correlated model Hamiltonian (hoppings, screened Coulomb integrals), density-functional theory within the LDA was used. ${ }^{9}$ The combined LDA+DMFT computational scheme was successfully applied to a wide range of compounds with degenerate (or almost degenerate) orbitals (for more details, see Ref. 10). In these cases the noninteracting LDA DOS was used to obtain the Green function of the system through a Hilbert transformation. Furthermore, the screened Coulomb interaction parameters $U$ and $J$ were calculated by constrained LDA. ${ }^{33}$

Quite generally, this scheme needs to be improved in two respects: (i) instead of the LDA DOS an LDA Hamiltonian with a few, relevant orbitals should be used to calculate the Green function, and (ii) a feedback from DMFT to LDA should be incorporated. Both of these problems are solved by the approach proposed in this work. In this method the Hamiltonian matrix in the WF basis set $H_{n n^{\prime}}^{W F}(\mathbf{k})$ is calculated from the LDA Hamiltonian via the projection procedure (6) and (23).
In DMFT the lattice problem becomes an effective singlesite problem which has to be solved self-consistently for the matrix self-energy $\hat{\Sigma}$ and the local matrix Green function

$$
G_{n n^{\prime}}(\varepsilon)=\frac{1}{V_{B Z}} \int d \mathbf{k}\left(\left[\left(\varepsilon+E_{f}^{(N)}\right) \hat{1}-\hat{H}^{W F}(\mathbf{k})-\hat{\Sigma}^{W F}(\varepsilon)\right]^{-1}\right)_{n n^{\prime}} .
$$

The integration can actually be restricted to the irreducible part of the Brillouin Zone (BZ) via the analytical tetrahedron method $^{38}$ with a subsequent symmetrization of the matrix Green function. The chemical potential $E_{f}^{(N)}$ is determined by the number of electrons on the $N$ interacting orbitals of interest. $^{35}$

The DMFT is based on the fact that in the $d=\infty$ limit the self-energy is local. ${ }^{36,37}$ Its matrix $\Sigma_{n n^{\prime}}(\varepsilon)\left(n, n^{\prime}\right.$ are WF indices) is defined in WF basis (3). If the trial functions in Eqs. (6) and (28) are chosen as the basis functions of the irreducible representation of the point symmetry group of some particular real system, ${ }^{39}$ the matrix Green function (27) and hence the matrix self-energy (3) can be made diagonal ${ }^{40}$ in the $n$ index for on-site matrix elements. However, the symmetry may be so low that the matrix self-energy is strongly off-diagonal. In this case one needs to employ a general formalism which is dealing with off-diagonal matrix Green functions and self-energies. ${ }^{34}$ In the present work we investigate systems $\left(\mathrm{SrVO}_{3}\right.$ and $\left.\mathrm{V}_{2} \mathrm{O}_{3}\right)$ where the symmetry is high enough to result in a diagonal matrix Green function and self-energy. For this reason we use a DMFT computational scheme $\mathrm{e}^{10}$ assuming diagonal matrices.

The DMFT single-site problem may be formulated as a self-consistent single-impurity Anderson model. ${ }^{6}$ The corresponding local one-particle matrix Green function $\hat{G}$ can be written as a functional integral ${ }^{6}$ involving an action where the Hamiltonian of the correlation problem under investigation, including the interaction term with the Hubbard interaction and Hund's rule couplings, enters. ${ }^{10}$ The action depends on the bath matrix Green function $\hat{\mathcal{G}}$ through

$$
(\hat{\mathcal{G}})^{-1}=(\hat{G})^{-1}+\hat{\Sigma} .
$$

To solve the functional integral of the effective single impurity Anderson problem, various methods can be used: quantum Monte Carlo (QMC), numerical renormalization group (NRG), exact diagonalization (ED), noncrossing approximation (NCA), etc. (for a brief overview of the methods see Ref. 10).

\section{Converting back to the full-orbital Hilbert space}

The matrix self-energy $\hat{\Sigma}^{W F}(\varepsilon)$ obtained as a solution of DMFT in Sec. II C is defined in the WF basis set (3). In order to compute the interacting Green function in the fullorbital Hilbert space (26) and (27) one has to convert it back to the full-orbital (LMTO) basis set. This can be easily done by using the linear-expansion form of the WFs in terms of the full-orbital basis set (15) and (17), 


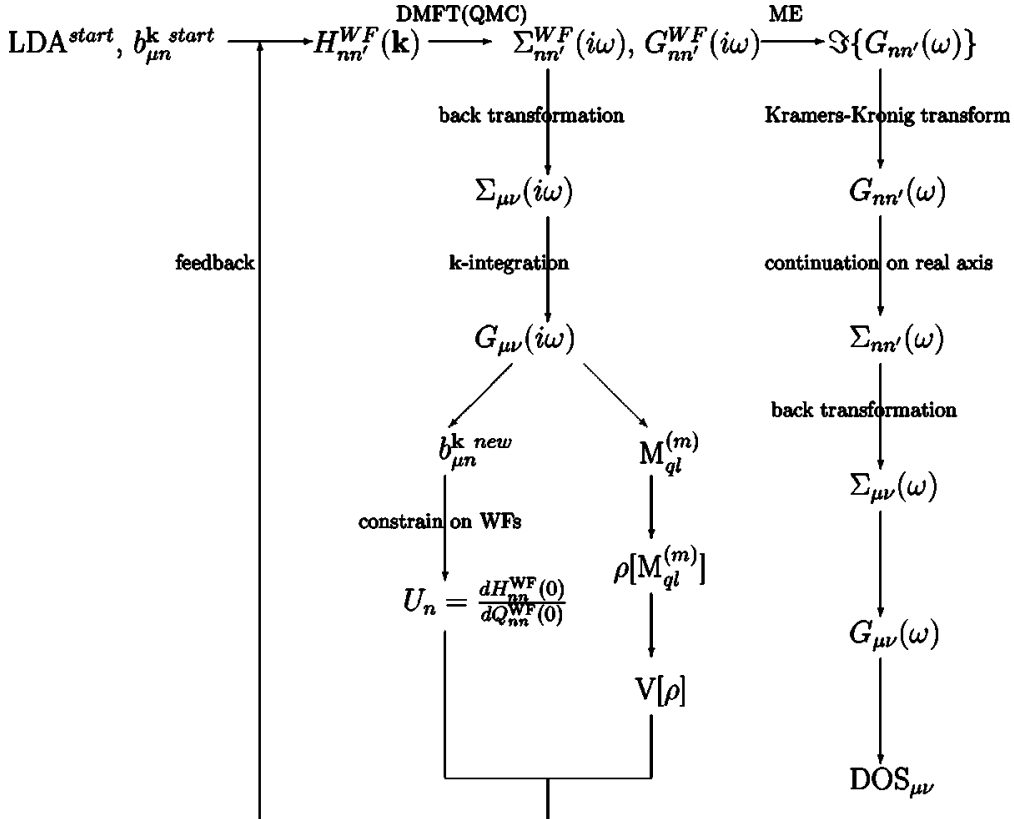

$$
\begin{aligned}
\sum_{\mu \nu}^{\mathbf{k}}(\varepsilon) & =\left\langle\phi_{\mu}^{\mathbf{k}}|\hat{\Sigma}(\varepsilon)| \phi_{\nu}^{\mathbf{k}}\right\rangle \\
& =\sum_{n}\left\langle\phi_{\mu}^{\mathbf{k}} \mid W_{n \mathbf{k}}\right\rangle \Sigma_{n n^{\prime}}(\varepsilon)\left\langle W_{n^{\prime} \mathbf{k}} \mid \phi_{\nu}^{\mathbf{k}}\right\rangle \\
& =\sum_{n} b_{\mu n}^{\mathbf{k}} \Sigma_{n n^{\prime}}(\varepsilon) b_{\nu n^{\prime}}^{\mathbf{k}^{*}} .
\end{aligned}
$$

Here we use the local form of the matrix self-energy as obtained in DMFT, but the formalism can be easily generalized. In the following we refer to this matrix self-energy as the "full-orbital" self-energy.

The matrix elements of the self-energy $\Sigma_{\mu \nu}^{\mathbf{k}}(\varepsilon)$ [Eq. (39)] together with the noninteracting Hamiltonian matrix $H_{\mu \nu}^{\mathbf{k}}$ allow one to calculate the matrix Green function $G_{\mu \nu}^{\mathbf{k}}(\varepsilon)[\mathrm{Eq}$. (27)] and thus the full-orbital interacting Green function $G\left(\mathbf{r}, \mathbf{r}^{\prime}, \varepsilon\right)\left[\right.$ Eq. (26)]. $G\left(\mathbf{r}, \mathbf{r}^{\prime}, \varepsilon\right)$ contains the full information about the system, and various electronic, magnetic, and spectral properties can be obtained from it. In Sec. III B we use the full-orbital interacting Green function computed within DMFT (QMC) to calculate the photoemission and $\mathrm{X}$-ray-absorption spectra for the strongly correlated vanadium oxides $\mathrm{SrVO}_{3}$ and $\mathrm{V}_{2} \mathrm{O}_{3}$, and to compare them with new bulk-sensitive experimental spectra.

One can also calculate the charge-density distribution modified by correlation effects via

$$
\rho(\mathbf{r})=-\frac{1}{\pi} \operatorname{Im} \int_{-\infty}^{E_{F}} d \varepsilon G(\mathbf{r}, \mathbf{r}, \varepsilon) .
$$

With this $\rho(\mathbf{r})$ one can recalculate the LDA potential (which is a functional of electron density). From the full-orbital Green function (26) one can recalculate new WFs (28) and (29) which together with the new LDA Hamiltonian allows one to obtain new parameters for the noninteracting Hamiltonian (24). With Eq. (25) one can then compute a new Coulomb interaction parameter $U$. The set of new LDA potential, WFs, and Coulomb interaction parameters calculated from
FIG. 1. Scheme of the $a b$ initio fully selfconsistent LDA+DMFT scheme based on the WF formalism (see text). the interacting Green function (26) defines the input for the next iteration step and hence closes the self-consistency loop in the proposed computation scheme. For the feedback from DMFT to LDA in the particular case of the LMTO method ${ }^{11}$ one needs a set of moments for the partial densities of states $M_{q l}^{(m)}$ for every atomic sphere $q$ and the orbital moment $l,{ }^{41}$ in order to calculate the new charge density and hence the new LDA potential:

$$
\begin{gathered}
M_{q l}^{(m)}=\int_{-\infty}^{E_{F}} d \varepsilon \varepsilon^{m} N_{q l}(\varepsilon), \\
N_{q l}(\varepsilon)=-\frac{1}{\pi N} \operatorname{Im} \sum_{\mathbf{k}} \sum_{m} G_{q l m, q l m}^{\mathbf{k}}(\varepsilon) .
\end{gathered}
$$

\section{E. Summary of the WF scheme}

For clarity, in Fig. 1 the essential steps of the WF scheme presented here are summarized. There are four interconnected parts in this scheme: (i) the basis of WF, (ii) the matrix elements of the Hamiltonian and the self-energy in the WF basis, (iii) the Coulomb interaction between electrons on the WFs, and (iv) the projection into the few-orbital basis and back transformation to the full-orbital basis which retains the information about all orbitals. First the matrix elements of the noninteracting Hamiltonian in reciprocal space $H_{n n^{\prime}}^{W F}(\mathbf{k})[\mathrm{Eq} .(23)]$ and the interaction term $\Sigma_{n n^{\prime}}(i \omega)$ [Eq. (3)] are written in the basis of explicitly defined WFs $\left|W_{n \mathbf{k}}\right\rangle[\mathrm{Eq} .(15)]$. The actual correlation problem, defined by the sum of these two terms (37), is then solved within the LDA+DMFT (QMC) approach. ${ }^{10}$ The local self-energy $\Sigma_{n n^{\prime}}(i \omega)$ obtained thereby is then transformed back from the Wannier basis to the full-orbital space (see Sec. II D). Furthermore, with the full-orbital self-energy $\Sigma_{\mu \nu}(i \omega)$ [Eq. (39)] the full-orbital Green function $G_{\mu \nu}(i \omega)$ [Eqs. (26) and (27)] for the correlated electrons is calculated by a $\mathbf{k}$ integration 
over the Brillouin zone. ${ }^{38}$ The Green function allows one to determine a new charge-density distribution (40) [in the LMTO case see Eq. (41)] and a new set of WFs [via Eqs. (28) and (29)]. This is used to construct a new LDA potential and new noninteracting Hamiltonian. Together with the new Coulomb interaction parameters $U_{n}$ [Eq. (25)] they serve as the input for the next iteration of calculations, thus completing the self-consistency loop. It should be stressed that in this scheme self-consistency involves not only the self-energy but also the basis of WFs in which it is defined, the charge density and LDA potential used for constructing the noninteracting Hamiltonian, and the interaction strength between electrons in the WFs.

After convergency is reached the maximum entropy method (MEM) (Ref. 46) can be used to obtain imaginary part of Green function $G_{n n^{\prime}}(i \omega)$. Then, using the KramersKronig transformation, the Green function on the real axis $G_{n n^{\prime}}(\omega)$ is computed. With that one can construct the WFs basis self-energy $\Sigma_{n n^{\prime}}(\omega)$ (see Appendix B). By the back transformation of $\Sigma_{n n^{\prime}}(\omega)$ with a subsequent $\mathbf{k}$ integration the full-orbital Green function $G_{\mu \nu}(\omega)$ is obtained, which now contains information not only about the states for which correlations are considered, but also for all other orbitals of the system. $G_{\mu \nu}(\omega)$ is used to obtain orbitally resolved spectral functions. This allows one, for example, to investigate the influence of the correlated orbitals (e.g., partially filled $t_{2 g}$ orbitals of $\mathrm{V}$ in $\mathrm{SrVO}_{3}$ and $\mathrm{V}_{2} \mathrm{O}_{3}$ ) on other orbitals (oxygen $2 p$ and vanadium $e_{g}$ orbitals). It also makes possible the computation of spectral functions in a wide energy region and not only in the vicinity of the Fermi level.

\section{RESULTS AND DISCUSSION}

In our earlier LDA+DMFT calculation scheme the specific properties of a material entered only via the LDA partial densities of states (DOSs) for the orbitals of interest. This procedure is valid for systems where the bands of interest are degenerate (as in cubic crystals; see Appendix A). For more complicated systems with lower symmetry one needs to employ the scheme proposed in this work, where the noninteracting Hamiltonian $\hat{H}^{W F}(\mathbf{k})$ [Eq. (23)] (projected on WFs) describing the $N$ orbitals under consideration is used for the calculation of $G_{n n^{\prime}}(\varepsilon)$ [Eq. (37)] within DMFT (see Sec. II C).

In this section we present results of LDA+DMFT calculations using the projected Hamiltonian $\hat{H}^{W F}(\mathbf{k})$. The scheme was applied to $\mathrm{SrVO}_{3}$ which has a cubic perovskite crystal structure, and to the trigonally distorted $\mathrm{V}_{2} \mathrm{O}_{3}$ (both in the insulating and metallic phase). The results are compared with previous LDA+DMFT calculations where the Hilbert transformation of the LDA DOSs was used for $\mathrm{SrVO}_{3}$ (Ref. 42) (see Sec. III A). A similar scheme was used by Liebsch ${ }^{43}$ to compute surface properties of $\mathrm{SrVO}_{3}$. One should note that the DOSs used for DMFT calculations of $\mathrm{V}_{2} \mathrm{O}_{3}$ were obtained by the TB-LMTO-ASA code version $47,{ }^{11}$ in contrast to Ref. 44 where the DOSs were calculated by the ASW method ${ }^{45}$ However, the DOSs obtained in both methods are very similar and do not produce much different LDA + DMFT results.
The full-orbital calculation scheme proposed in this work allows one to answer an important question: how do Coulomb correlations between some orbitals affect the other orbitals, and in particular, how does the interaction between the partially filled $t_{2 g}$ orbitals of the V $3 d$ shell influence the filled $\mathrm{O}-2 p$ and the unoccupied $\mathrm{V}-3 d\left(e_{g}^{\sigma}\right)$ bands? To answer these questions the matrix of the self-energy $\Sigma_{n n^{\prime}}(\varepsilon)(3)$ was converted back to the full-orbital basis set from the WF basis, and the full-orbital interacting Green function (27) was calculated. From that, total and partial DOSs were computed to produce theoretical spectra for comparison with the experimental photoemission and x-ray-absorption data.

In this work, QMC simulations ${ }^{8}$ were used to solve the effective single impurity Anderson problem in the DMFT loop. The result of the DMFT (QMC) calculation is the selfenergy on the imaginary energy axis $\Sigma_{n n^{\prime}}(i \omega)$. To find the full-orbital self-energy $\Sigma_{\mu \nu}^{\mathbf{k}}(\varepsilon)$ on the real energy axis one has to perform an analytical continuation. The procedure is described in Appendix B. A self-consistent computation of the charge density as described in section II D was not yet performed. Investigations of correlation effects on the unoccupied cubic $e_{g}^{\sigma}$ states for both $\mathrm{SrVO}_{3}$ and $\mathrm{V}_{2} \mathrm{O}_{3}$ are also a matter of future calculations.

\section{A. Comparison of DMFT results obtained by Hilbert transformation of the LDA DOS and the projected Hamiltonian $\hat{H}^{W F}(\mathbf{k})$}

The cubic perovskite $\mathrm{SrVO}_{3}$ can serve as a test case for our method. For the cubic $O_{h}$ point symmetry group, the three $3 d$ orbitals $x y, x z, y z$ transform according to the triply degenerate $t_{2 g}$ irreducible representation of this group. Hence the corresponding Green function and self-energy matrices have diagonal form with equal diagonal elements. As shown in Appendix A, the results of the Hilbert transformation using LDA DOS must coincide with the results of the procedure of integration over the Brillouin zone with the projected $3 \times 3 t_{2 g}$ Hamiltonian.

We use the same LDA DOS and interaction parameters for $\mathrm{SrVO}_{3}$ as in our previous papers. ${ }^{42}$ The $\mathrm{V}-3 d\left(t_{2 g}\right)$ states form a partially filled band in $\mathrm{SrVO}_{3}$. All $t_{2 g}$ orbitals $(x y, x z, z y)$ are equivalent due to the cubic symmetry of the lattice, so only the results for one of the $t_{2 g}$ orbitals are presented in Fig. 2. In this figure $\mathrm{V}-3 d\left(t_{2 g}\right)$ spectral functions, calculated using the LDA DOS and the projected Hamiltonian are shown. It is easy to see that both results are almost identical. The small differences between these two curves are due to the MEM (Ref. 46) used for the calculation of the spectral function on the real energy axis from the DMFT Green function.

We also applied our Hamiltonain scheme to a more complicated system with lower symmetry where one can expect deviations from the results obtained using the LDA DOS. We performed LDA+DMFT (QMC) calculations for the insulating and metallic phases of $\mathrm{V}_{2} \mathrm{O}_{3}$ with the projected $3 \times 3 t_{2 g}$ Hamiltonian and several $U$ values (unless stated otherwise the values of $U$ are the same as in Ref. 44). Here and in the following we use an exchange Coulomb parameter $J$ $=0.93 \mathrm{eV}$ for $\mathrm{V}_{2} \mathrm{O}_{3} \cdot{ }^{44}$ In contrast to $\mathrm{SrVO}_{3}, \mathrm{~V}_{2} \mathrm{O}_{3}$ has a non- 


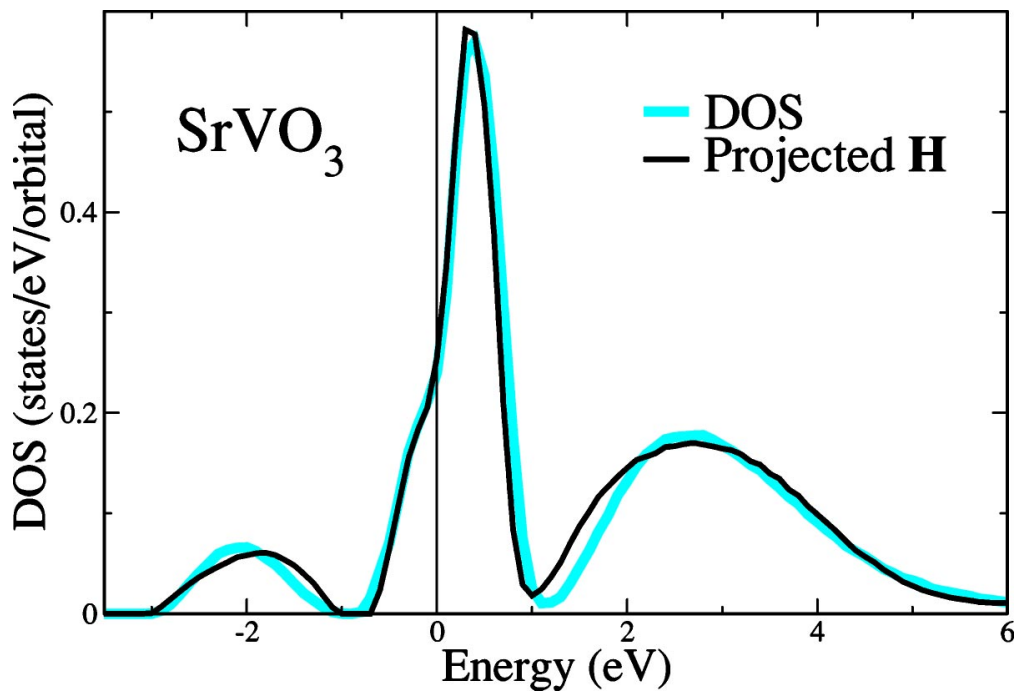

FIG. 2. Comparison of $\mathrm{V}-3 d\left(t_{2 g}\right)$ spectral functions for $\mathrm{SrVO}_{3}$ calculated via LDA+DMFT (QMC) using: LDA DOS—light line; projected Hamiltonian-black line. The Fermi level corresponds to zero.

cubic trigonal symmetry ( space group $R \overline{3} c$ ). Therefore we use the basis functions of the trigonal $D_{3 d}$ point group instead of the cubic $O_{h}$ group. In this basis set the V-3d shell is split into two groups of bands. The three $t_{2 g}$ bands are located around the Fermi energy, the two degenerate $e_{g}^{\sigma}$ bands are at higher energies. Interesting to us are the partially filled $t_{2 g}$ bands which are formed by one $a_{1 g}$ and two degenerate $e_{g}^{\pi}$ orbitals.

In Fig. 3, we present $\mathrm{V}-3 d\left(t_{2 g}\right)$ spectral functions resulting from DOS (LMTO and ASW) and Hamiltonian calculations at $U=4.5 \mathrm{eV}$, averaged over the three $t_{2 g}$ bands. The spectrum computed with ASW input is taken from Ref. 44.

As mentioned before, the differences between the curves calculated with ASW and LMTO DOS as input are small; there are only minor deviations in the peak height. In the comparison between the DOS calculations and the Hamiltonian calculation, it is interesting to note that for these averaged spectra, the differences are relatively small. We find the typical four peak structure (with lower Hubbard, quasiparticle peak, and double-peaked upper Hubbard band split by Hund's rule coupling) for all three calculations. Only the position and the height of the quasiparticle peak are quite different for the Hamiltonian calculation, indicating a more insulating solution than for the DOS calculations at the same $U$ value. The similarity between the results for the DOS and Hamiltonian calculations is not surprising, because the trigonal distortion in $\mathrm{V}_{2} \mathrm{O}_{3}$ is relatively small. Therefore the center of gravity and the bandwidth of the three $t_{2 g}$ bands do not change much and the DOS calculations can still produce accurate results.

The differences between DOS and Hamilton calculations are more pronounced when one compares the band-resolved $t_{2 g}$ spectra. In Figs. 4 and 5, the $a_{1 g}$ and $e_{g}^{\pi}$ spectra for the insulating and metallic crystal structure are presented for $U$ $=4.5,5.0$, and $5.5 \mathrm{eV}$.

Here the curves calculated by different schemes are distinctively different. This is especially clear from the upper part of Figs. 4 and 5 corresponding to the $a_{1 g}$ orbital of the $\mathrm{V}-3 d\left(t_{2 g}\right)$ subband. This is due to the fact that the hybridization effects are better accounted for by the projected Hamiltonian, which includes not only intraband hoppings but also interband on-site and intersite hoppings. The latter ef-

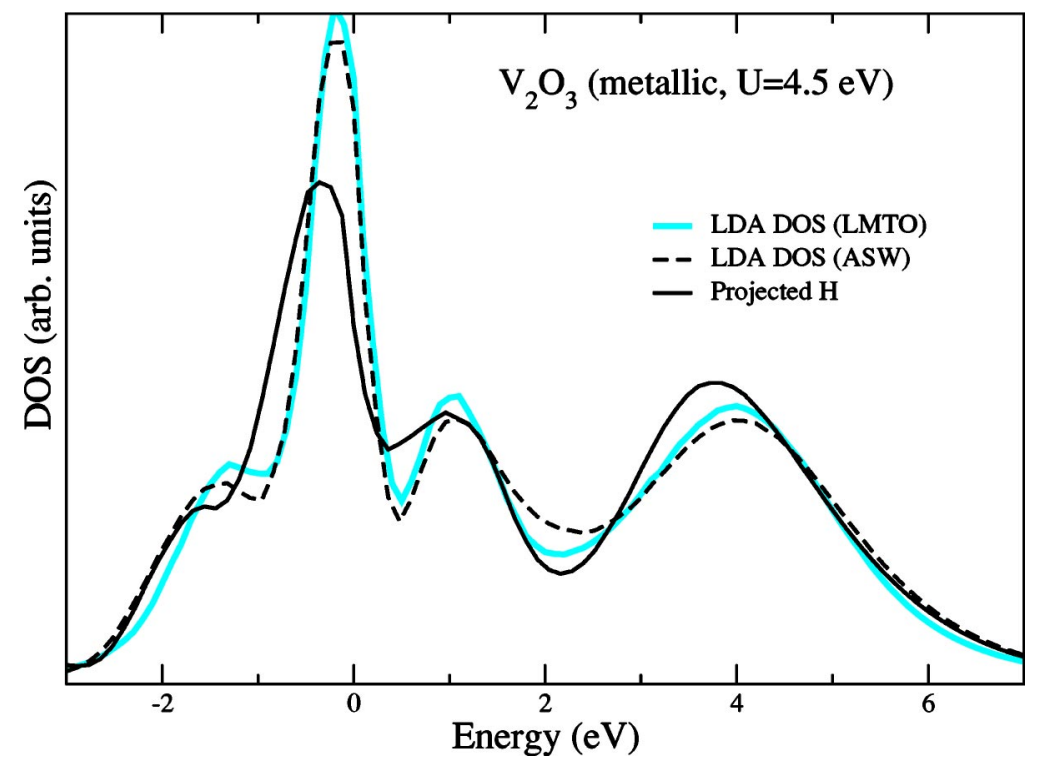

FIG. 3. Comparison of $\mathrm{V}-3 d\left(t_{2 g}\right)$ spectral functions for $\mathrm{V}_{2} \mathrm{O}_{3}$ in the metallic phase calculated via LDA+DMFT (QMC) using: LDA DOS (LMTO)_light line (Ref. 44); LDA DOS (ASW)_-dashed line; projected Hamiltonianblack line. The Fermi level corresponds to zero. 


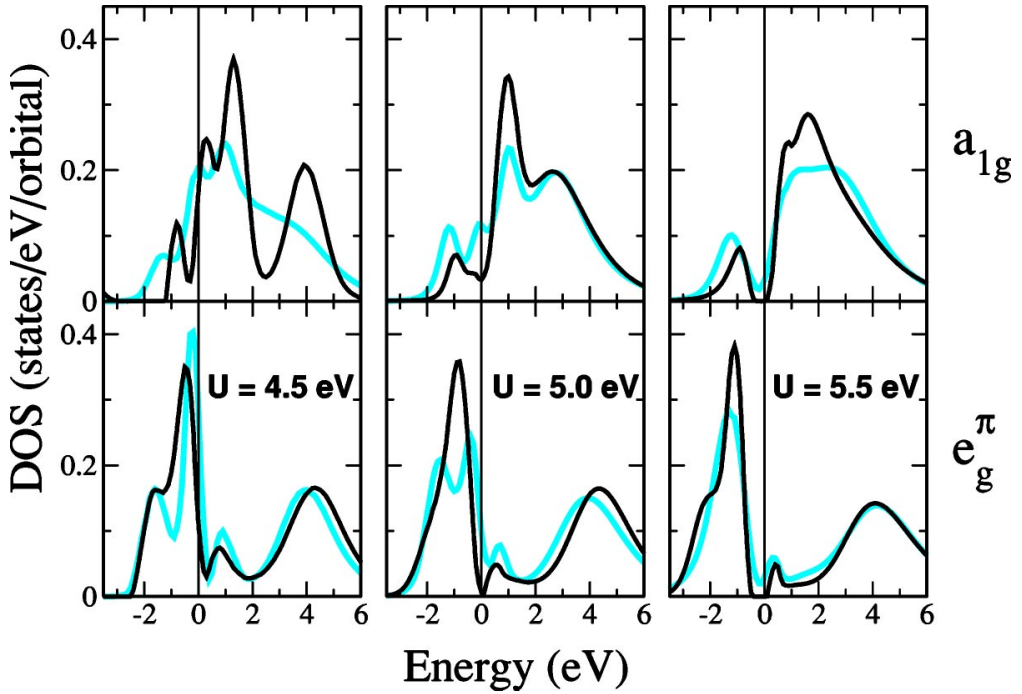

FIG. 4. Comparison of V-3d(t $\left.t_{2 g}\right)$ spectral functions for $\mathrm{V}_{2} \mathrm{O}_{3}$ in the insulating phase calculated via LDA+DMFT (QMC) using: LDA DOS_light line; projected Hamiltonian-black line. Upper figures $-a_{1 g}$; lower figures $-e_{g}^{\pi}$ orbitals. The Fermi level corresponds to zero. fectively increases the bandwidth of the $a_{1 g}$ orbital. The corresponding spectral functions have a different peak structure and different intensities.

Another conclusion from this series of figures is that the results obtained with the Hamiltonian procedure show a more insulating behavior for the same values of $U$ than the results with the scheme using the LDA DOS.

\section{B. Results of full-orbital calculations: Comparison of calculated spectra with experimental photoemission and $x$-ray-absorption data}

The full-orbital calculations scheme proposed in this work produces an interacting Green function $G\left(\mathbf{r}, r^{\prime}, \varepsilon\right)$ [Eq. (26)]. The knowledge of the full Green function allows us to calculate the spectral function not only for the states with correlations $\left[\mathrm{V}-3 d\left(t_{2 g}\right)\right.$ orbitals in the case of $\mathrm{SrVO}_{3}$ and $\mathrm{V}_{2} \mathrm{O}_{3}$, but also their effect on the lower-lying occupied oxygen $2 p$ states and the higher-lying unoccupied $\mathrm{V}-3 d\left(e_{g}^{\sigma}\right)$ states, thus facilitating a comparison of the calculated and experimental spectra in a wide energy region.
Valence-band photoemission spectroscopy (PES) using high photon energies and $\mathrm{O}-1 s \rightarrow 2 p$ x-ray-absorption spectroscopy (XAS) were performed on the beamline BL25SU at the SPring-8 synchrotron radiation facility. The PES spectra were taken using a GAMMADATA-SCIENTA SES-200 electron energy analyzer and XAS spectra were measured by the total electron yield. The overall energy resolution was set to $0.2 \mathrm{eV}$. The pressure in the analyzer chamber was about $4 \times 10^{-8} \mathrm{~Pa}$. Single crystals of $\mathrm{SrVO}_{3}$ were cooled to $20 \mathrm{~K}$ and clean surfaces were obtained by fracturing in situ for the PES spectra, and by scraping in situ for the much more bulk sensitive XAS spectra. Well-oriented single-crystalline $\mathrm{V}_{2} \mathrm{O}_{3}$ samples were cleaved in situ at a temperature near the metalinsulator transition, yielding clean specular surfaces. The surface cleanliness was confirmed before and after the spectral run.

We start with the results for the $\mathrm{SrVO}_{3}$ system. In Fig. 6, the self-energy on the real energy axis for $\mathrm{SrVO}_{3}$ calculated via Eq. (B2) is shown. The $\mathrm{m}^{*} / \mathrm{m}$ ratio is calculated via $m^{*} / m=1 /\{1-[\partial \operatorname{Re} \Sigma(\omega) / \partial \omega]\}$. This self-energy was used for the calculation of total and partial DOSs in the full-orbital Hilbert space (see Figs. 7 and 8). In Fig. 7, the total spectral

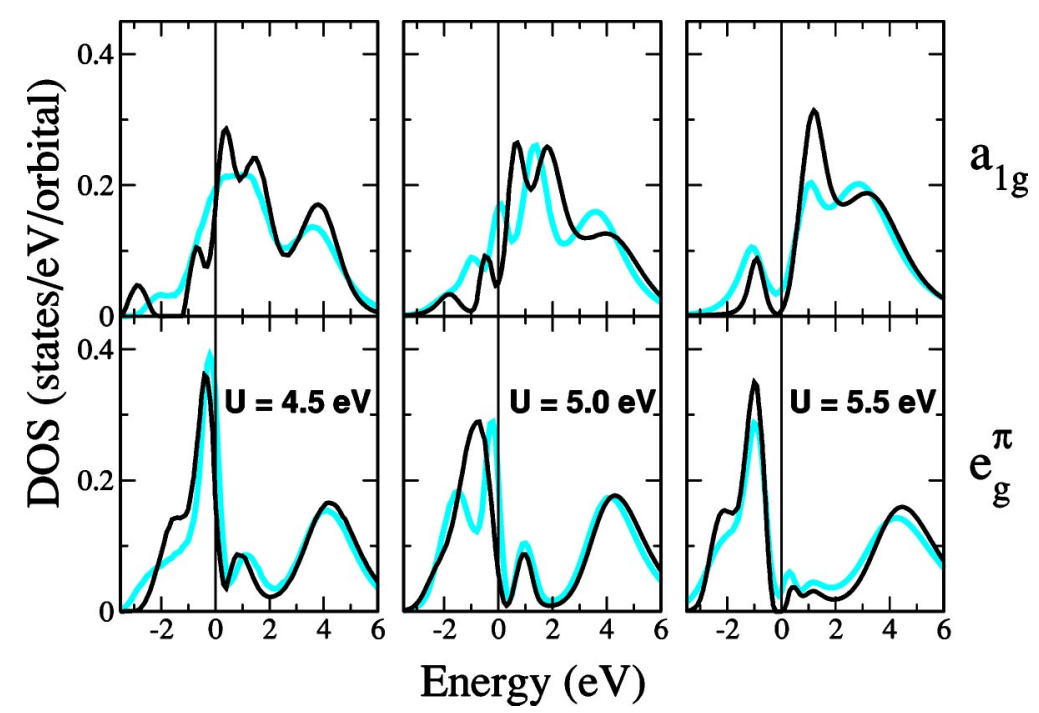

$125119-10$
FIG. 5. Comparison of $\mathrm{V}-3 d\left(t_{2 g}\right)$ spectral functions for $\mathrm{V}_{2} \mathrm{O}_{3}$ in the metal phase calculated via LDA+DMFT (QMC) using: LDA DOSlight line; projected Hamiltonian-black line. Upper figures $-a_{1 g}$; lower figures $-e_{g}^{\pi}$ orbitals. The Fermi level corresponds to zero. 


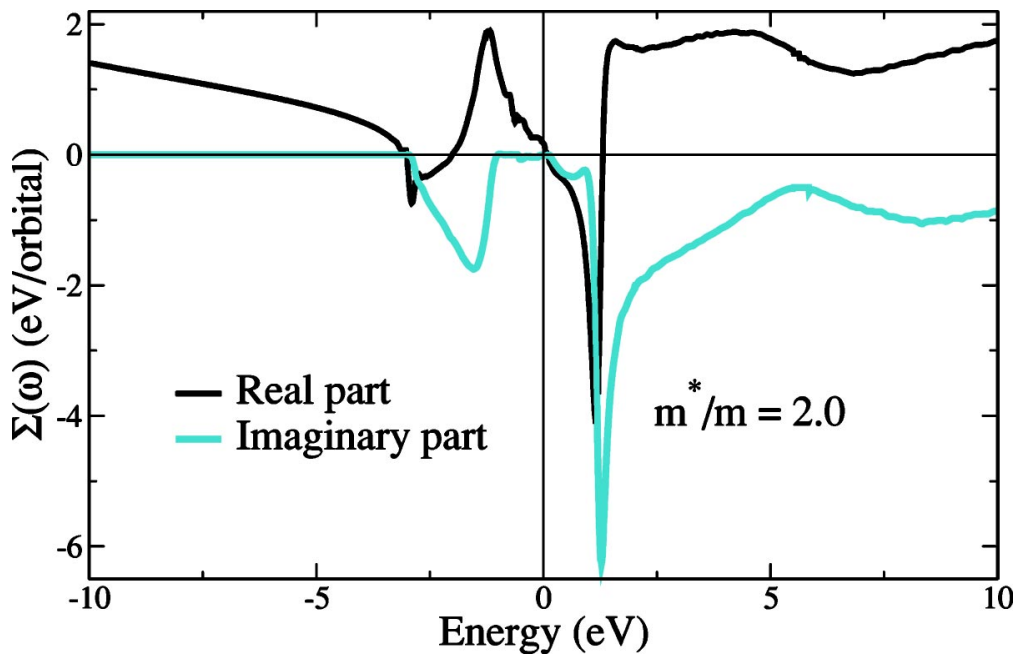

FIG. 6. Self-energy on real energy axis for $\mathrm{SrVO}_{3}$. Real part-full black line; imaginary part-full light line. The Fermi level corresponds to zero.

functions of $\mathrm{SrVO}_{3}$ are presented. Differences between LDA and full-orbital LDA+DMFT (QMC) spectral functions mainly occur near the Fermi level. In particular, the LDA spectral function has a more pronounced quasiparticle peak near $1 \mathrm{eV}$. The DOS calculated using the full-orbital selfenergy (see Fig. 8) has a three-peak structure: lower Hubbard band from -4 to -2 (suppressed by oxygen states), quasiparticle peak and upper Hubbard band located at about $3 \mathrm{eV}$. The origin of the upper Hubbard band becomes clear from Fig. 8. One can see that this broad peak is the sum of V$3 d\left(e_{g}^{\sigma}\right)$ and V-3d $\left(e_{g}^{\sigma}\right)$ states. It should be noted that correlations on the $\mathrm{V}-3 d\left(e_{g}^{\sigma}\right)$ orbitals were not explicitly included here. We find, however, that the $\mathrm{V}-3 d\left(e_{g}^{\sigma}\right)$ states are slightly modified by the full-orbital self-energy due to the hybridization with the correlated $\mathrm{V}-3 d\left(e_{g}^{\sigma}\right)$ orbitals. The question of how correlations affect the position and width of $\mathrm{V}-3 d\left(e_{g}^{\sigma}\right)$ states directly can only be answered by employing the full $3 d$-shell $5 \times 5$ projected Hamiltonian.

Introducing correlations between $t_{2 g}$ states changes significantly the total and partial (is not shown here directly but can be conjectured from Fig. 9) LDA DOSs of $\mathrm{SrVO}_{3}$. The main modification is a transfer of spectral weight from the energy region near the Fermi level to the lower and upper Hubbard bands, and the reduction of the weight of the qua- siparticle peak. Our calculations suggest a strongly correlated but still metallic ground state for $\mathrm{SrVO}_{3}$.

To compare our results with the experimental PES we calculated a weighted sum of $\mathrm{V}-3 d$ and $\mathrm{O}-2 p$ spectral functions according to the photoemission cross section ratio $3: 1$, corresponding to an experimental photon energy $900 \mathrm{eV}$. The theoretical spectra were multiplied with the Fermi function corresponding to $20 \mathrm{~K}$ and broadened with a $0.2-\mathrm{eV}$ Gaussian to take into account the instrumental resolution. In Fig. 9 one can see that the full-orbital spectra obtained in this way describe not only the quasiparticle peak, but also the peak at $-6 \mathrm{eV}$ and the shoulder at $-3.5 \mathrm{eV}$ of the PES spectra. Since previous LDA+DMFT (QMC) results were taking into account only the $t_{2 g}$ states they were not able to describe PES spectra below $-2 \mathrm{eV}$. It is interesting to note that previous experimental studies did not find any states at -3.5 $\mathrm{eV}$; only the new spectra reported here show this feature. The agreement between the experimental and theoretical spectra is expected to improve when $e_{g}$ states are included in the correlation problem and when also the charge and LDA potential self-consistency is fully implemented.

The influence of correlation effects on the electronic structure will be more pronounced for systems close to the Mott insulator transition. For this purpose we compare the total and partial DOS's for $\mathrm{V}_{2} \mathrm{O}_{3}$ in the insulating and me-

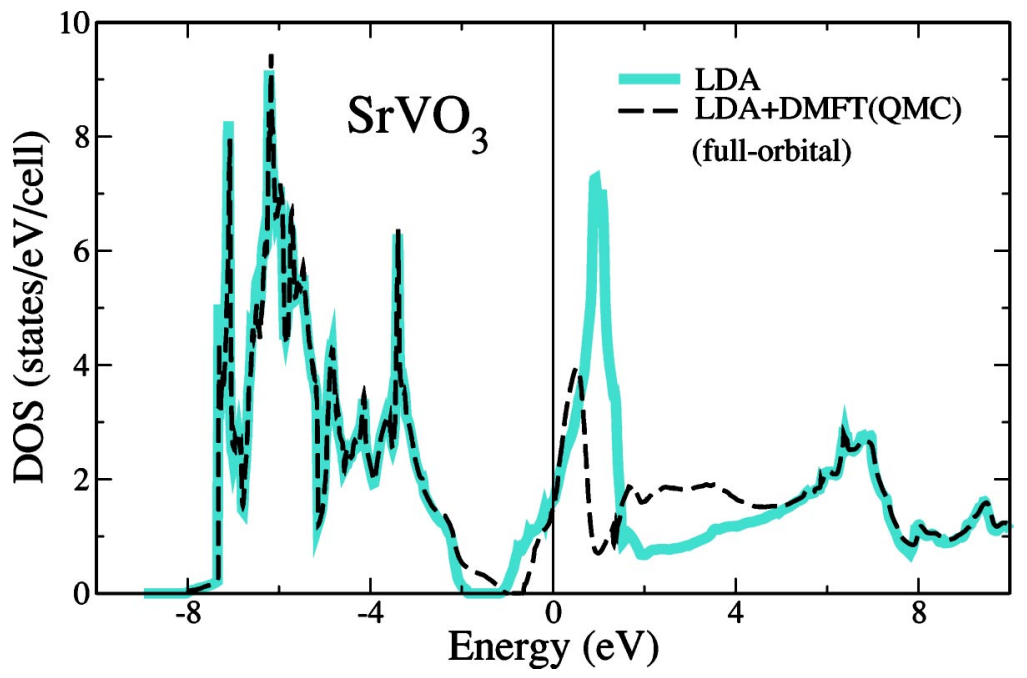

FIG. 7. Comparison of total spectral functions of $\mathrm{SrVO}_{3}$ calculated via: LDA-full light line; using the full-orbital self-energy from LDA +DMFT (QMC)—dashed black line. The Fermi level corresponds to zero. 


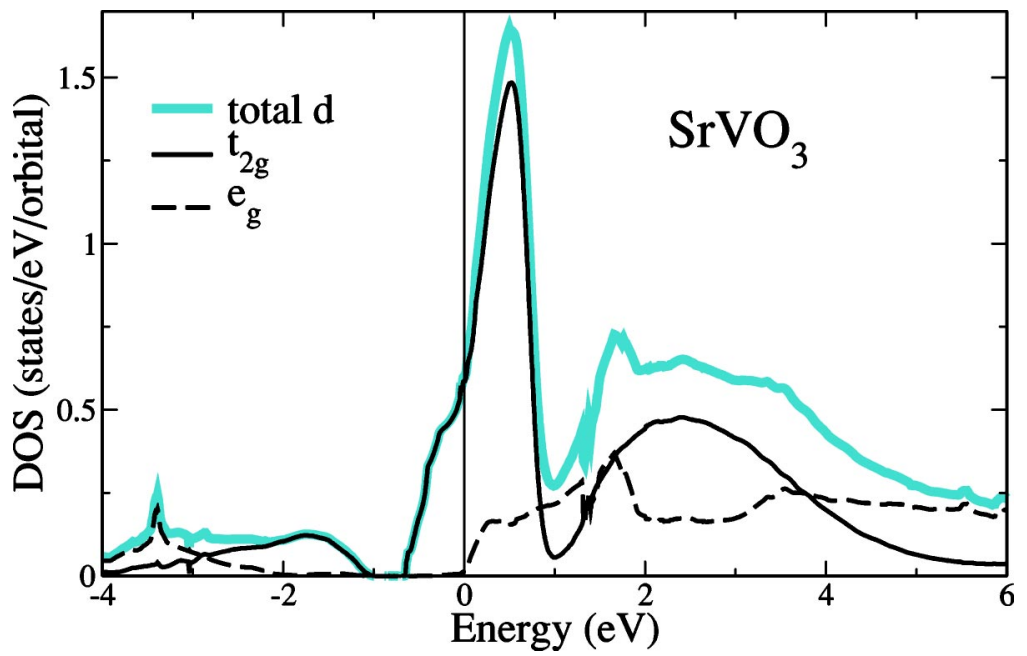

FIG. 8. Partial V-3d spectral functions for $\mathrm{SrVO}_{3}$ calculated using the full-orbital selfenergy from LDA+DMFT (QMC). Total $d$-full light line; $t_{2 g}$-full black line; $e_{g}$-dashed black line. The Fermi level corresponds to zero.

tallic phases calculated via LDA and full-orbital LDA+DMFT ${ }^{48,49}$ Since the V-3d $\left.t_{2 g}\right)$ orbitals in trigonal $\mathrm{V}_{2} \mathrm{O}_{3}$ split into nonequivalent $a_{1 g}$ and $e_{g}^{\pi}$ states, the selfenergy will be different for these orbitals (see Fig. 10).

In Fig. 11 one can see that the introduction of correlation effects changes the total DOS drastically. Whereas the LDA DOS is metallic for both metallic and insulating phases, the LDA+DMFT spectra clearly show the metal-insulator transition. Although the change near the Fermi level is the most prominent, modifications between -3 to $-7 \mathrm{eV}$ are also seen. Moreover, there are also changes in the $e_{g}^{\sigma}$ states which are caused by the hybridization with the correlated $t_{2 g}$ bands, and are not due to a direct calculation of correlations in the $e_{g}^{\sigma}$ states. In Fig. 12 the total $d$-state DOS above the Fermi energy, consisting of $t_{2 g}$ and $e_{g}^{\sigma}$ states, is shown.

In Fig. 13 a comparison of experimental PES and calculated LDA+DMFT (QMC) $\left(t_{2 g}\right.$ only and full-orbital) spectra is presented. The full-orbital spectrum is a weighted sum of

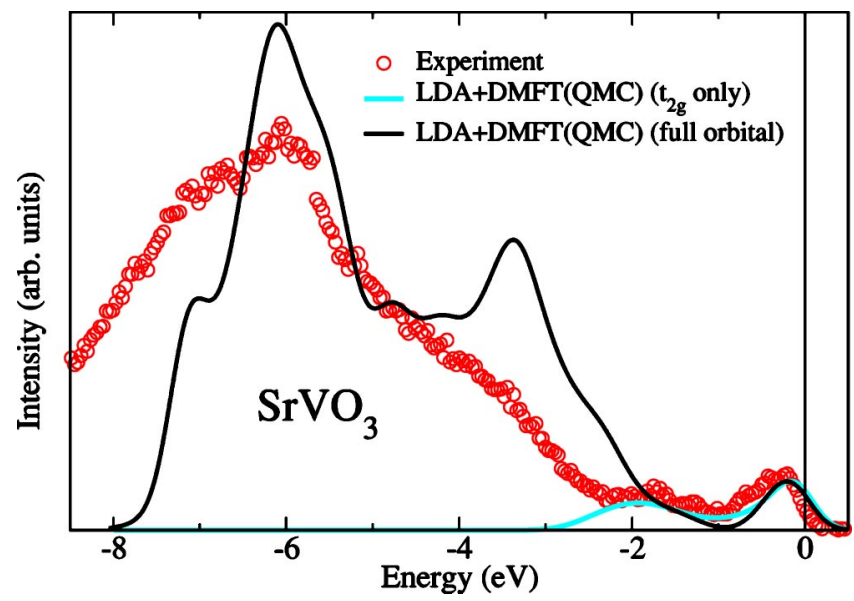

FIG. 9. Comparison of photoemission spectra of $\mathrm{SrVO}_{3}$ with spectral functions calculated via LDA+DMFT (QMC): taking into account only $t_{2 g}$-light line; using full-orbital self-energy-black line. Theoretical spectra are multplied with the Fermi function corresponding to $20 \mathrm{~K}$ and broadened with a $0.2-\mathrm{eV}$ Gaussian to account for the instrumental resolution. Intensities are normalized to yield the same height for the quasiparticle peaks. The Fermi level corresponds to zero. the calculated $\mathrm{V}-3 d$ and $\mathrm{O}-2 p$ spectral functions, according to the photoemission cross-section ratio $2: 1$, corresponding to the experimental photon energy of $500 \mathrm{eV}$. The theoretical spectra were multiplied with the Fermi function and broadened with a Gaussian of $0.2 \mathrm{eV}^{47}$ Below the Fermi energy the LDA+DMFT (QMC) spectral functions (for $t_{2 g}$ only and the full-orbital scheme) agree quite well with the PES spectra. However, the theory curves do not yet describe fine details. For example, the PES spectrum of the insulator phase shows two definite slope changes, at roughly -0.6 and -1.5 $\mathrm{eV}$, producing a rather flat topped spectrum centered on -1 $\mathrm{eV}$, whereas the theory curves show a single peak centered on $-1 \mathrm{eV}$.

A comparison of the calculated LDA+DMFT (QMC) full-orbital spectral functions and the $\mathrm{O} 1 s \rightarrow 2 p$ XAS spectrum is shown on Fig. 14. The full-orbital spectra (which are the partial $\mathrm{O}-2 p$ spectra for the unoccupied $\mathrm{V}-3 d$ states) are

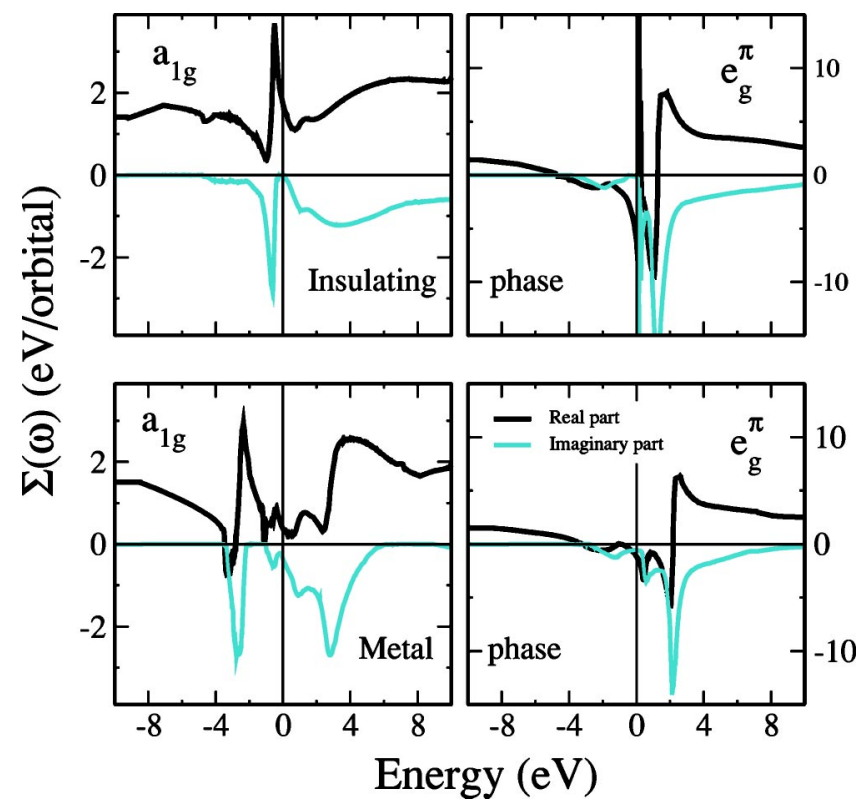

FIG. 10. Self-energy on real energy axis for $\mathrm{V}_{2} \mathrm{O}_{3}$. Real part of the self-energy from LDA+DMFT (QMC) - full black line; imaginary part-full light line. The Fermi level corresponds to zero. 


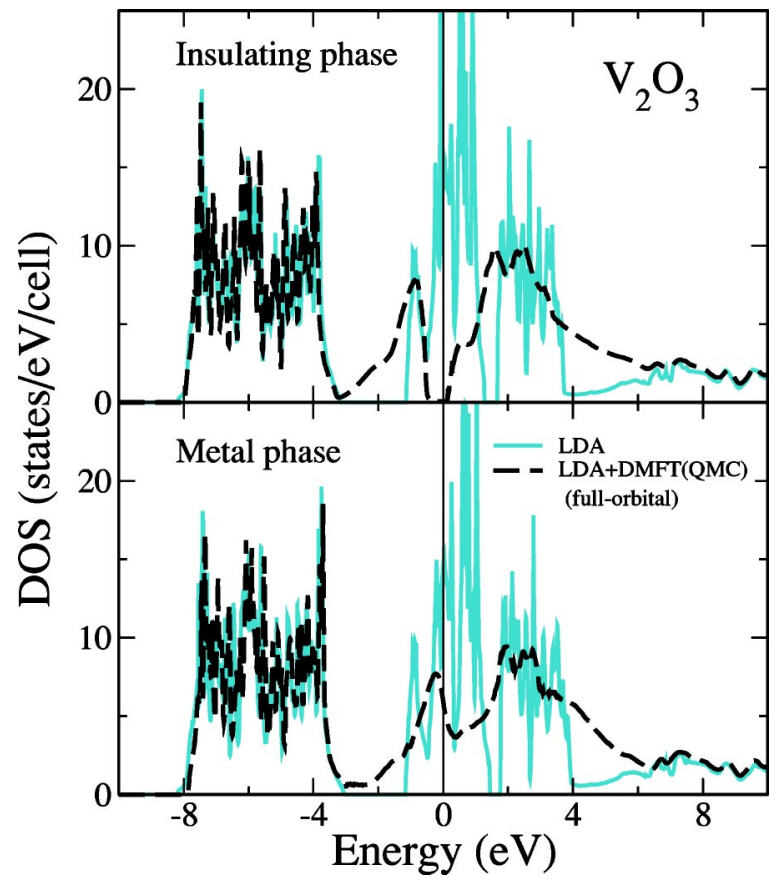

FIG. 11. Comparison of total spectral functions of $\mathrm{V}_{2} \mathrm{O}_{3}$ calculated via: LDA - full light line; using full-orbital self-energy from LDA+DMFT (QMC)—dashed black line. The Fermi level corresponds to zero.

found to agree with the experimental spectrum as to the relative weights of the part nearest the Fermi energy and the parts at higher energy. This is due to the inclusion of $\mathrm{O}-2 p$ and V-3d $\left(e_{g}^{\sigma}\right)$ states in the calculations. The strong hybrid-

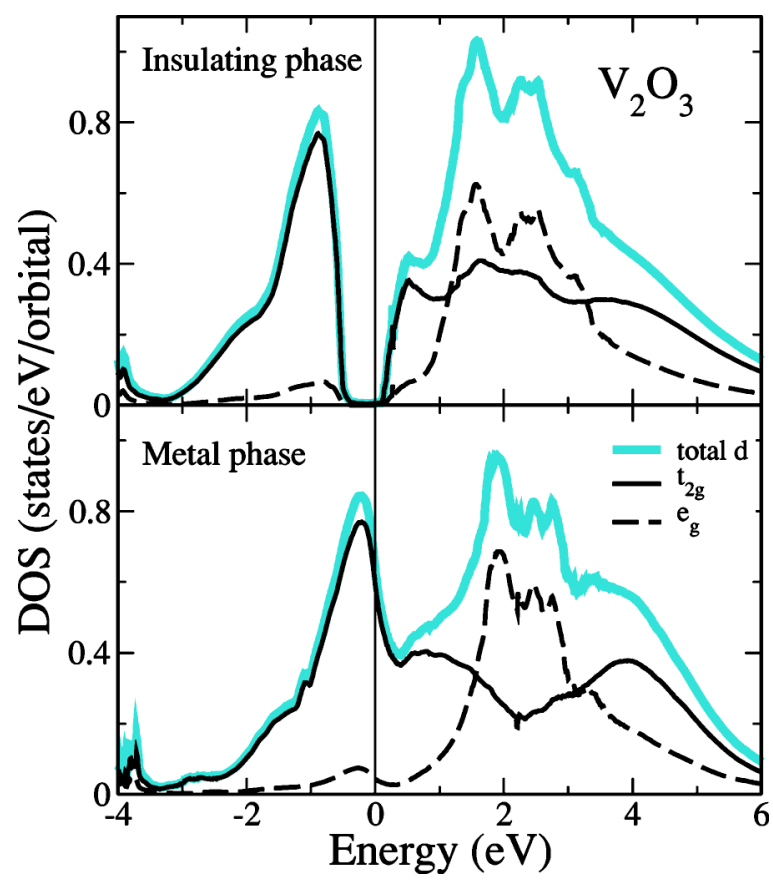

FIG. 12. Partial V-3d spectral functions for $\mathrm{V}_{2} \mathrm{O}_{3}$ calculated using full-orbital self-energy from LDA+DMFT (QMC). Total $d$-full light line; $t_{2 g}$-full black line; $e_{g}$-dashed black line. The Fermi level corresponds to zero.

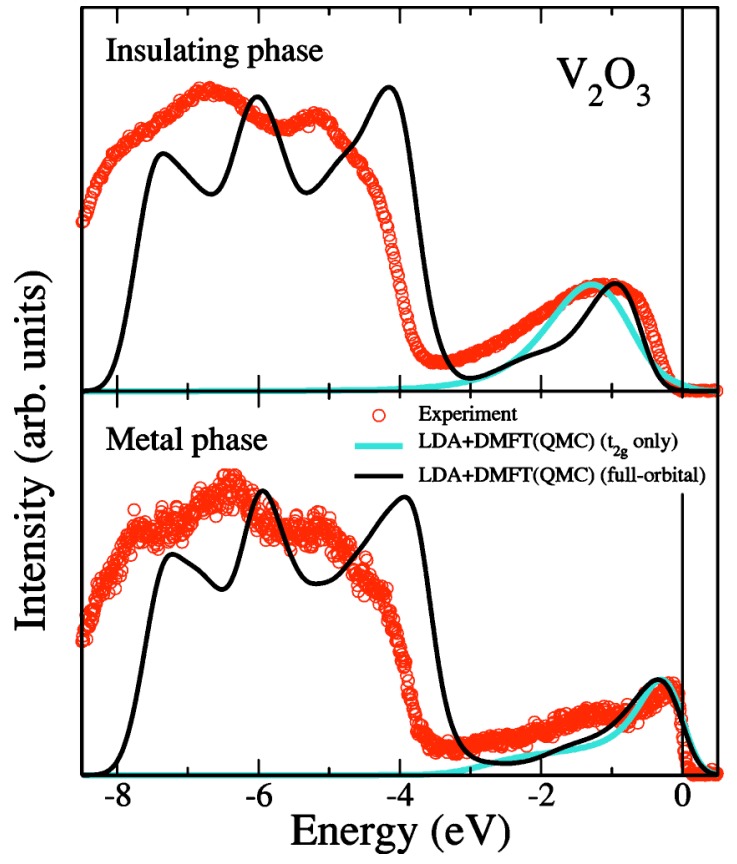

FIG. 13. Comparison of photoemission spectra of $\mathrm{V}_{2} \mathrm{O}_{3}$ with spectral functions calculated via LDA+DMFT (QMC): taking into account only $t_{2 g}$-light line; using full-orbital self-energy-black line. Theoretical spectra are multiplied with the Fermi function and broadened with a $0.2-\mathrm{eV}$ Gaussian to simulate instrumental resolution. Intensities are normalized for peaks situated around $-1 \mathrm{eV}$. The Fermi level corresponds to zero.

ization of the O-2p with the $d$ states is described more correctly in the full-orbital calculations and the inclusion of $e_{g}^{\sigma}$ states (even without correlations) significantly changes the $\mathrm{V}-3 d$ spectrum in the energy region above the Fermi edge. The inclusion of correlations in the $e_{g}^{\sigma}$ states within a $5 \times 5$ projected V-3d Hamiltonian is expected to add spectral weight in the energy interval between 3 and $5 \mathrm{eV}$ (see Fig. 14).

\section{CONCLUSION}

We formulated a fully $a b$ initio and self-consistent computational scheme based on Wannier functions (WFs) for the calculation of the electronic structure of strongly correlated compounds. The WF formalism provides an explicit strategy for the construction of the matrix elements of the required operators (Hamiltonian, self-energy, etc.), both in full-orbital and few-orbital bases, in real and reciprocal representations. The WF formalism allows one to project these operators from the full-orbital space to the few-orbital space and back, keeping the complete information about all orbitals. These projections are the essential ingredients of the computational scheme presented here. The self-consistency involves not only the self-energy but also the WF basis itself, the charge density with the LDA potential and the interaction strength parameters between the electrons on the WFs. The spectra obtained thereby found to be in good agreement with new bulk-sensitive experimental data.

In the present work we did not yet employ the full scheme (see Fig. 1) to investigate spectral functions of strongly cor- 


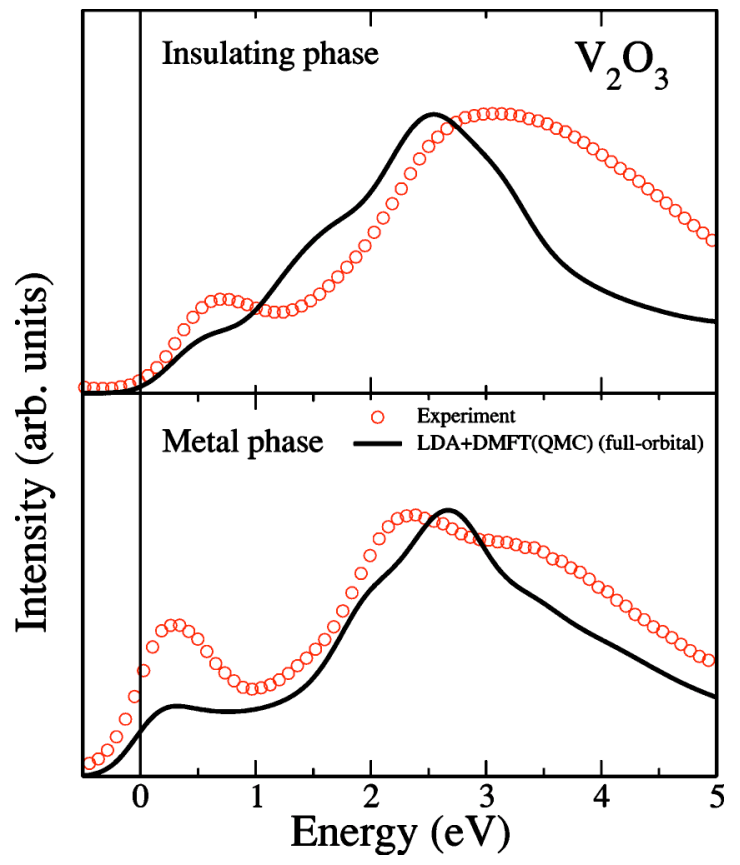

FIG. 14. Comparison of O- $1 s \rightarrow 2 p$ x-ray-absorption spectroscopy (XAS) spectra of $\mathrm{V}_{2} \mathrm{O}_{3}$ with spectral functions calculated via LDA+DMFT (QMC) using full-orbital self-energy. Theoretical spectra are multiplied with the Fermi function and broadened with a 0.2-eV Gaussian to simulate instrumental resolution. Intensities of the theory curves are normalized to the correct number of $\mathrm{V}-3 d$ electrons in the unit cell (eight electrons). The experimental curve is normalized to the same peak height as the full orbital curve. The Fermi level corresponds to zero.

related systems but used only few-orbital Hamiltonians with $t_{2 g}$ symmetry. Clearly, full $d$-shell DMFT (QMC) results including $e_{g}$ states will provide additional information about correlation effects in the system. Such studies, as well as constrained LDA calculations in the WF basis and investigations of the feedback from the DMFT to the LDA part, are in progress now.

\section{ACKNOWLEDGMENT}

This work was supported by Russian Basic Research Foundation Grant Nos. RFFI-GFEN-03-02-39024-a, RFFI04-02-16096, by the joint UrO-SO Project No. N22, by the U.S. NSF Grant No. DMR-03-02825, and at POSTECH by KOSEF through eSSC and by the Deutsche Forschungsgemeinschaft through Sonderforschungsbereich 484. I.N. acknowledges support from the Grant of President of Russian Federation for young scientists (Grant No. MK-95.2003.02), Dynasty Foundation, and International Center for Fundamental Physics in Moscow program for young scientists 2004), Russian Science Support Foundation program for young Ph.D. of Russian Academy of Science 2004. We would like to acknowledge John von Neumann Institut für Computing, Jülich for providing and support of computing time and Japan Synchrotron Radiation Research Institute for support of soft x-ray-absorption and photoemission studies on SPring-8.

\section{APPENDIX A: HILBERT TRANSFORMATION}

For cubic systems the matrix of the self-energy (for example, for $t_{2 g}$ orbitals) is diagonal and all diagonal elements are equal. Therefore the calculation of the Green function within DMFT by integration of the Hamiltonian over the BZ is equivalent to the Hilbert transformation of the noninteracting (LDA) DOS $N^{0}(\epsilon)$ :

$$
\begin{aligned}
\Sigma(\omega) & =\left(\begin{array}{cccc}
\sigma(\omega) & 0 & \ldots & 0 \\
0 & \sigma(\omega) & \ldots & 0 \\
\vdots & \vdots & \ddots & \vdots \\
0 & 0 & \ldots & \sigma(\omega)
\end{array}\right) \Rightarrow G(\omega) \\
& =\int_{I B Z}\left[\omega-\Sigma(\omega)-H_{L D A}^{0}(k)\right]^{-1} d k \\
& =G^{0}[\omega-\sigma(\omega)] \\
& =\int \frac{N^{0}(\varepsilon)}{\omega-\sigma(\omega)-\epsilon} d \epsilon .
\end{aligned}
$$

\section{APPENDIX B: SELF-ENERGY ON THE REAL ENERGY AXIS}

DMFT produces Green functions and self-energies on the imaginary energy axis. With the maximum entropy method, ${ }^{46}$ the spectral function on the real energy axis is calculated, which yields the imaginary part of the Green function. In order to obtain the self-energy on the real energy axis, the full complex Green function $G(\varepsilon)$ is calculated using its imaginary part obtained by MEM:

$$
G(\varepsilon)=-\frac{1}{\pi} \int_{-\infty}^{\infty} \frac{\operatorname{Im} G\left(\varepsilon^{\prime}\right) d \varepsilon^{\prime}}{\varepsilon-\varepsilon^{\prime}+i \eta}
$$

The self-energy for real energies is then calculated by solving the following two equations with the two variables $\operatorname{Re} \Sigma(\varepsilon)$ and $\operatorname{Im} \Sigma(\varepsilon)$ :

$$
\operatorname{Re}, \operatorname{Im}\{G(\varepsilon)\}=\operatorname{Re}, \operatorname{Im}\left\{\int_{B Z}[\varepsilon-H(k)-\Sigma(\varepsilon)]^{-1} d k\right\},
$$

where

$$
\Sigma(\varepsilon)=\operatorname{Re} \Sigma(\varepsilon)+i \operatorname{Im} \Sigma(\varepsilon) .
$$

\section{APPENDIX C: NONORTHOGONAL BASIS SET}

Equation (11) is valid only for orthogonal LMTO orbitals. In the case of general nonorthogonal LMTOs (or any other atomic-type orbital basis set), an orthogonalization procedure can be used by defining an orthogonal Hamiltonian $\widetilde{H}$ and the corresponding eigenvectors $\widetilde{C}$ for the nonorthogonal Hamiltonian $H$ and overlapping matrix $O$ : 


$$
\begin{gathered}
\widetilde{H}=O^{-1 / 2} H O^{-1 / 2}, \\
\widetilde{C}=O^{1 / 2} C .
\end{gathered}
$$

This orthogonalization is equivalent to the basis set transformation:

$$
\left|\widetilde{\phi}_{n}\right\rangle=\sum_{n^{\prime}} O_{n n^{\prime}}^{-1 / 2}\left|\phi_{n^{\prime}}\right\rangle
$$

Then for the nonorthogonal LMTO the trial function $\left|\phi_{n}\right\rangle$ has to be replaced by $\left|\widetilde{\phi}_{n}\right\rangle$ and the eigenvectors with coefficients $c_{j i}^{\mathbf{k}}$ by $\widetilde{c}_{j i}^{\mathbf{k}}$.
${ }^{1}$ G. H. Wannier, Phys. Rev. 52, 191 (1937).

${ }^{2}$ N. Marzari and D. Vanderbilt, Phys. Rev. B 56, 12847 (1997); N. Marzari, I. Souza, and D. Vanderbilt, Psi-k Newsletter 57, 112 (2003) [psi-k.dl.ac.uk/newsletters/News_57/Highlight_57.pdf].

${ }^{3}$ Wei Ku, H. Rosner, W. E. Pickett, and R. T. Scalettar, Phys. Rev. Lett. 89, 167204 (2002).

${ }^{4}$ D. Vollhardt, in Correlated Electron Systems, edited by V. J. Emery (World Scientific, Singapore, 1993), p. 57.

${ }^{5}$ Th. Pruschke, M. Jarrell, and J. K. Freericks, Adv. Phys. 44, 187 (1995).

${ }^{6}$ A. Georges, G. Kotliar, W. Krauth, and M. J. Rozenberg, Rev. Mod. Phys. 68, 13 (1996).

${ }^{7}$ G. Kotliar and D. Vollhardt, Phys. Today 57 (3), 53 (2004).

${ }^{8}$ J. E. Hirsch and R. M. Fye, Phys. Rev. Lett. 56, 2521 (1986); M. Jarrell, ibid. 69, 168 (1992); M. Rozenberg, X. Y. Zhang, and G. Kotliar, ibid. 69, 1236 (1992); A. Georges and W. Krauth, ibid. 69, 1240 (1992); M. Jarrell, in Numerical Methods for Lattice Quantum Many-Body Problems, edited by D. Scalapino, (Addison-Wesley, Reading, MA, 1997).

${ }^{9}$ V. I. Anisimov, A. I. Poteryaev, M. A. Korotin, A. O. Anokhin, and G. Kotliar, J. Phys.: Condens. Matter 9, 7359 (1997); A. I. Lichtenstein and M. I. Katsnelson, Phys. Rev. B 57, 6884 (1998)

${ }^{10}$ K. Held, I. A. Nekrasov, N. Blümer, V. I. Anisimov, and D. Vollhardt, Int. J. Mod. Phys. B 15, 2611 (2001); K. Held, I. A. Nekrasov, G. Keller, V. Eyert, N. Blümer, A. K. McMahan, R. T. Scalettar, T. Pruschke, V. I. Anisimov, and D. Vollhardt, in Quantum Simulations of Complex Many-Body Systems: From Theory to Algorithms, edited by J. Grotendorst, D. Marks, and A. Muramatsu, NIC Series No.10 (NIC Directors, Forschunszentrum Jülich, 2002), p. 175-209; K. Held, I. A. Nekrasov, G. Keller, V. Eyert, N. Blumer, A. K. McMahan, R. T. Scalettar, Th. Pruschke, V. I. Anisimov, and D. Vollhardt, Psi-k Newsletter 56, 65 (2003) [psi-k.dl.ac.uk/newsletters/News_56/ Highlight_56.pdf]; A. I. Lichtenstein, M. I. Katsnelson, G. Kotliar, in Electron Correlations and Materials Properties, 2nd ed., edited by A. Gonis, Nicholis Kioussis, and Mikael Ciftan (Kluwer Academic/Plenum, New York, 2002), p. 428.

${ }^{11}$ O. K. Andersen, Phys. Rev. B 12, 3060 (1975); O. Gunnarsson, O. Jepsen, and O. K. Andersen, ibid. 27, 7144 (1983).

${ }^{12}$ L. F. Mattheiss and D. R. Hamann, Phys. Rev. B 33, 823 (1986).

${ }^{13}$ The self-energy is, in general, a frequency-dependent, nonlocal quantity which describes the effect of interactions on the noninteracting Hamiltonian. Here it enters into the theory as the difference between the inverse matrix Green functions of the bath and the impurity, Eq. (38).

${ }^{14}$ General properties of the Wannier functions have been investigated in Refs. 15-20 and 27-29.
${ }^{15}$ G. F. Koster, Phys. Rev. 89, 67 (1953).

${ }^{16}$ G. Parzen, Phys. Rev. 89, 237 (1953).

${ }^{17}$ P. O. Löwdin, J. Chem. Phys. 18, 365 (1950).

${ }^{18}$ G. G. Hall, Philos. Mag. 43, 338 (1952).

${ }^{19}$ R. I. Blount, in Solid State Physics, edited by F. Seitz and D. Turnbull (Academic, New York, 1962), Vol. 13, p. 305.

${ }^{20}$ W. Kohn, Phys. Rev. 115, 809 (1959).

${ }^{21}$ W. Kohn and J. R. Onffroy, Phys. Rev. B 8, 2485 (1973).

${ }^{22}$ W. Kohn, Phys. Rev. B 7, 4388 (1973).

${ }^{23}$ W. Kohn, Phys. Rev. B 10, 382 (1974).

${ }^{24}$ J. J. Gehr and W. Kohn, Phys. Rev. B 10, 448 (1974).

${ }^{25}$ M. R. Geller and W. Kohn, Phys. Rev. B 48, 14085 (1993).

${ }^{26}$ P. W. Anderson, Phys. Rev. Lett. 21, 13 (1968).

${ }^{27}$ J. Des Cloizeaux, Phys. Rev. 129, 554 (1963).

${ }^{28}$ J. Des Cloizeaux, Phys. Rev. 135, A685 (1964).

${ }^{29}$ J. Des Cloizeaux, Phys. Rev. 135, A698 (1964).

${ }^{30}$ I. Schnell, G. Czycholl, and R. C. Albers, Phys. Rev. B 68, 245102 (2003).

${ }^{31}$ O. K. Andersen and T. Saha-Dasgupta, Phys. Rev. B 62, R16 219 (2000).

${ }^{32}$ Z. V. Pchelkina, D. E. Kondakov, I. V. Leonov, V. I. Anisimov, V. A. Gavrichkov, and S. G. Ovchinnikov, Phys. Met. Metallogr. (to be published).

${ }^{33}$ O. Gunnarsson, O. K. Andersen, O. Jepsen, and J. Zaanen, Phys. Rev. B 39, 1708 (1989); V. I. Anisimov and O. Gunnarsson, ibid. 43, 7570 (1991).

${ }^{34}$ E. Pavarini, S. Biermann, A. Poteryaev, A. I. Lichtenstein, A. Georges, and O. K. Andersen, Phys. Rev. Lett. 92, 176403 (2004).

${ }^{35}$ In the calculations performed in the present paper we assumed the position of the Fermi level to be unchanged when going from LDA to DMFT (QMC). It was found in previous investigations (Ref. 10) that this is a good approximation for vanadates.

${ }^{36}$ W. Metzner and D. Vollhardt, Phys. Rev. Lett. 62, 324 (1989).

${ }^{37}$ E. Müller-Hartmann, Z. Phys. B: Condens. Matter 74, 507 (1989); , Z. Phys. B: Condens. Matter 76, 211 (1989).

${ }^{38} \mathrm{Ph}$. Lambin and J. P. Vigneron, Phys. Rev. B 29, 3430 (1984); O. Jepsen and O. K. Andersen, Solid State Commun. 9, 1763 (1971); P. E. Blöchl, O. Jepsen, and O. K. Andersen, Phys. Rev. B 49, 16223 (1994).

${ }^{39}$ For example, the $t_{2 g}$ and $e_{g}$ orbitals of the $d$ shell $(N=5)$ in the case of the $O_{h}$ point group of symmetry for a cubic lattice.

${ }^{40}$ Or nearly diagonal, if the symmetry is lowered by a relatively small distortion of the crystal structure.

${ }^{41}$ H. L. Skriver, The LMTO Method, Springer Series on Solid State Science. No. 41 (Springer, NewYork, 1984).

${ }^{42}$ I. A. Nekrasov, G. Keller, D. E. Kondakov, A. V. Kozhevnikov, Th. Pruschke, K. Held, D. Vollhardt, and V. I. Anisimov, cond- 
mat/0211508; A. Sekiyama, H. Fujiwara, S. Imada, H. Eisaki, S. I. Uchida, K. Takegahara, H. Harima, Y. Saitoh, and S. Suga cond-mat/0206471; A. Sekiyama, H. Fujiwara, S. Imada, S. Suga, H. Eisaki, S. I. Uchida, K. Takegahara, H. Harima, Y. Saitoh, I. A. Nekrasov, G. Keller, D. E. Kondakov, A. V. Kozhevnikov, Th. Pruschke, K. Held, D. Vollhardt, and V. I. Anisimov, Phys. Rev. Lett. 93, 156402 (2004).

${ }^{43}$ A. Liebsch, Phys. Rev. Lett. 90, 096401 (2003).

${ }^{44}$ K. Held, G. Keller, V. Eyert, V. I. Anisimov, and D. Vollhardt, Phys. Rev. Lett. 86, 5345 (2001).

${ }^{45}$ V. Eyert, Int. J. Quantum Chem. 77, 1007 (2000) (Special Issue: Electronic Structure of Materials, edited by M. Defranceschi).
${ }^{46}$ For a review on the maximum entropy method, see M. Jarrell and J. E. Gubernatis, Phys. Rep. 269, 133 (1996).

${ }^{47}$ S.-K. Mo, J. D. Denlinger, H.-D. Kim, J.-H. Park, J. W. Allen, A. Sekiyama, A. Yamasaki, K. Kadono, S. Suga, Y. Saitoh, T. Muro, P. Metcalf, G. Keller, K. Held, V. Eyert, V. I. Anisimov, and D. Vollhardt, Phys. Rev. Lett. 90, 186403 (2004).

${ }^{48}$ G. Keller, K. Held, V. Eyert, D. Vollhardt, and V. I. Anisimov, cond-mat/0402133.

${ }^{49}$ For illustration purposes we chose the most insulating ( $U$ $=5.5 \mathrm{eV}$ ) for insulating $\mathrm{V}_{2} \mathrm{O}_{3}$ and the most metallic solutions $(U=4.5)$ for metallic $\mathrm{V}_{2} \mathrm{O}_{3}$. 